\title{
Pervasive compartment-specific regulation of gene expression during homeostatic synaptic scaling
}

\section{Journal Article}

\section{Author(s):}

Colameo, David (D); Rajman, Marek; Soutschek, Michael; Bicker, Silvia; von Ziegler, Lukas; Bohacek, Johannes (D); Winterer, Jochen; Germain, Pierre-Luc (D); Dieterich, Christoph; Schratt, Gerhard

\section{Publication date:}

2021-10-05

\section{Permanent link:}

https://doi.org/10.3929/ethz-b-000502410

\section{Rights / license:}

Creative Commons Attribution 4.0 International

\section{Originally published in:}

EMBO Reports 22(10), https://doi.org/10.15252/embr.202052094

\section{Funding acknowledgement:}

172889 - Dissecting stress-induced molecular changes in circuits underlying anxiety (SNF) 


\title{
Pervasive compartment-specific regulation of gene expression during homeostatic synaptic scaling
}

\author{
David Colameo ${ }^{1,2, \dagger}$ (D), Marek Rajman ${ }^{3, \dagger, t}$, Michael Soutschek ${ }^{1,2, \dagger}$ D , Silvia Bicker ${ }^{1,2}$ (D), \\ Lukas von Ziegler ${ }^{2,4}$, Johannes Bohacek ${ }^{2,4}$, Jochen Winterer ${ }^{1,2}$ (D) , Pierre-Luc Germain ${ }^{5,6}$ (D), \\ Christoph Dieterich ${ }^{7}$ (D) \& Gerhard Schratt ${ }^{1,2, *}$ (D)
}

\begin{abstract}
Synaptic scaling is a form of homeostatic plasticity which allows neurons to adjust their action potential firing rate in response to chronic alterations in neural activity. Synaptic scaling requires profound changes in gene expression, but the relative contribution of local and cell-wide mechanisms is controversial. Here we perform a comprehensive multi-omics characterization of the somatic and process compartments of primary rat hippocampal neurons during synaptic scaling. We uncover both highly compartment-specific and correlating changes in the neuronal transcriptome and proteome. Whereas downregulation of crucial regulators of neuronal excitability occurs primarily in the somatic compartment, structural components of excitatory postsynapses are mostly downregulated in processes. Local inhibition of protein synthesis in processes during scaling is confirmed for candidate synaptic proteins. Motif analysis further suggests an important role for trans-acting post-transcriptional regulators, including RNA-binding proteins and microRNAs, in the local regulation of the corresponding mRNAs. Altogether, our study indicates that, during synaptic scaling, compartmentalized gene expression changes might co-exist with neuron-wide mechanisms to allow synaptic computation and homeostasis.
\end{abstract}

Keywords cellular compartment; homeostatic plasticity; local translation; microRNA; synaptic scaling

Subject Categories Methods \& Resources; Neuroscience

DOI 10.15252/embr.202052094 | Received 16 November 2020 | Revised 12 July

2021 | Accepted 20 July 2021 | Published online 16 August 2021

EMBO Reports (2021) 22: e52094

\section{Introduction}

Synaptic scaling is an important cellular mechanism that keeps excitatory synaptic strength in a physiological range in response to chronic ( $>6 \mathrm{~h}$ ) changes in synaptic input (Turrigiano, 2008). In the mammalian brain, synaptic scaling plays important roles during neural circuit development (e.g. activity-dependent development of the visual and barrel cortex) (Desai et al, 2002; Glazewski et al, 2017) and in cognition in the adult (e.g. memory consolidation during sleep) (Tononi \& Cirelli, 2014; Diering et al, 2017). Moreover, defects in synaptic scaling have been associated with the pathophysiology of several neurological diseases, e.g. autism (Rett syndrome), mental retardation (e.g. Fragile-X Syndrome) (Ramocki \& Zoghbi, 2008), epilepsy (Swann \& Rho, 2014) and mood disorders (Kavalali \& Monteggia, 2012). Mechanistically, synaptic scaling involves mainly two components (Turrigiano, 2008). First, a sensing mechanism for the detection of changes in neuronal firing rates, such as intracellular calcium-dependent pathways. Second, executing mechanisms which bring about changes in AMPA- and NMDAtype glutamate receptor function at excitatory synapses. Recent evidence suggests that synaptic scaling requires changes in gene expression, both at the level of mRNA transcription (Ibata et al, 2008; Schaukowitch et al, 2017) and translation (Goold \& Nicoll, 2010; Schanzenbacher et al, 2016; Schanzenbacher et al, 2018; Dorrbaum et al, 2020). This leads to a remodelling of the synaptic proteome, in particular by synthesis/degradation of neurotransmitter receptors, presynaptic proteins and components of the calciumdependent signalling pathways (Schanzenbacher et al, 2016; Rajman et al, 2017; Dorrbaum et al, 2020). The molecular mechanisms underlying these orchestrated changes in de novo protein synthesis, however, are only poorly understood. In hippocampal

\footnotetext{
1 Laboratory of Systems Neuroscience, Institute for Neuroscience, Department of Health Science and Technology, Swiss Federal Institute of Technology ETH, Zurich, Switzerland

2 Neuroscience Center Zurich, ETH Zurich and University of Zurich, Zurich, Switzerland

3 Institute for Physiological Chemistry, Biochemical-Pharmacological Center Marburg, Philipps-University of Marburg, Marburg, Cermany

4 Laboratory of Behavioural and Molecular Neuroscience, Institute for Neuroscience, Department of Health Science and Technology, Swiss Federal Institute of Technology ETH, Zurich, Switzerland

5 Institute for Neuroscience, Department of Health Science and Technology, Swiss Federal Institute of Technology ETH, Zurich, Switzerland

6 Laboratory of Statistical Bioinformatics, Department of Molecular Life Sciences, University of Zürich, Zurich, Switzerland

7 Section of Bioinformatics and Systems Cardiology, Department of Internal Medicine III and Klaus Tschira Institute for Integrative Computational Cardiology, University of Heidelberg, Heidelberg, Germany

*Corresponding author. Tel: +41 4463381 32; E-mail: gerhard.schratt@hest.ethz.ch

These authors contributed equally to this work

†Present address: Neuroscience Therapeutic Area, UCB Pharma, Braine l’Alleud, Belgium
} 
neurons, specific mRNAs are locally translated in the synaptodendritic compartment, which provides neurons with a means to regulate the activity of individual synapses or at least dendritic branches (Martin \& Ephrussi, 2009). Interestingly, in contrast to classical Hebbian synaptic plasticity, homeostatic plasticity was initially believed to operate exclusively at a global level, either at the level of entire neurons or networks (Ibata et al, 2008). However, theoretical considerations in the past challenged this view and suggested an important contribution of local mechanisms (e.g. operating at the level of individual dendritic segments) to synaptic scaling (Rabinowitch \& Segev, 2008). This view is supported by experimental results from cultured hippocampal neurons, which show local dendritic regulation of retinoic acid receptor signalling (Aoto et al, 2008) and AMPA-type glutamate receptor synthesis (Sutton et al, 2004; Sutton et al, 2006) in response to synaptic scaling up induced by chronic activity blockade. In contrast, whether synaptic downscaling in response to chronically elevated activity similarly involves local regulation of gene expression has not been addressed. More generally, compartmentalized changes in gene expression and their regulatory mechanisms during homeostatic plasticity or any other form of synaptic plasticity have not been comprehensively assessed on a more general level using multiomics approaches.

\section{Results}

In this study, we interrogated compartmentalized gene expression in rat hippocampal neurons undergoing homeostatic plasticity using a multi-omics approach. Therefore, we combined two in vitro model systems that we had previously established. First, a compartmentalized primary rat hippocampal neuron culture system, whereby neurons are plated on the upper side of filter inserts with small pore size $(1 \mu \mathrm{m})$ that selectively allow the growth of neuronal processes (axons, dendrites), but not somata, to the lower side of the inserts (Fig 1A) (Bicker et al, 2013). Second, a pharmacological stimulation protocol $(48 \mathrm{~h})$ treatment with the GABA-A receptor antagonist picrotoxin (PTX) that induces a robust and chronic increase in network activity followed by downscaling of excitatory synaptic strength (Fiore et al, 2014). Using patch-clamp recordings, we detected a significant reduction in amplitude of miniature excitatory postsynaptic currents (mEPSC) in hippocampal pyramidal neurons $48 \mathrm{~h}$ post PTX treatment, demonstrating robust downscaling of excitatory synapses (Figs $1 \mathrm{~B}$ and EV1A-C). To validate the compartmentalized culture system, we first performed a comparative transcriptomic analysis of the somatic and process compartment in unstimulated neurons using ribosome depletion RNA sequencing (ribo(-)RNAseq). Thereby, we identified 1,231/1,673 genes that were significantly enriched with a $\operatorname{logFC}$ of greater than \pm 1 in the somatic/process compartment, respectively (Fig 1C). As expected, many well-known dendritically and axonally localized mRNAs, e.g. Camk2a, Shank2/3, Homer1, Dlg4 and Syn1, were significantly enriched in the process compartment. In contrast, genes encoding nuclear proteins, such as Polr2c, Sfpq and Nono, were significantly enriched in the somatic compartment (Fig 1C). GO-term analysis indicates the overrepresentation of specific cellular components in these compartments: ribosomal subunits, postsynaptic density and mitochondrial respiratory chain complex in the process compartment: extracellular matrix, extracellular space and secretory granule lumen in the somatic compartment (Fig 1D; Appendix Fig S1). Importantly, we observed a very high correlation between our RNA-seq dataset and a recently published meta-analysis (von Kugelgen \& Chekulaeva, 2020) of neurite-enriched mRNAs derived from 11 independent high coverage datasets (Fig EV2A-E). Together, these results are consistent with previous large-scale studies (Poon et al, 2006; Cajigas et al, 2012; Zappulo et al, 2017; Kuzniewska et al, 2020) and suggest that our approach is able to faithfully capture differences in mRNA distribution between neuronal compartments.

We next investigated whether mRNA compartmentalization translated into corresponding changes in the neuronal proteome. Towards this aim, we prepared protein extracts from the somatic and process compartment of primary rat hippocampal neurons and subjected them to label-free proteomics (see Materials and Methods). In four independent preparations, we identified unique peptides corresponding to a total of 4,250 different proteins in the somatic/process compartment, demonstrating a high sensitivity of our approach despite the low amount of starting material. Principal component analysis (PCA) of the replicates indicates a high reproducibility of both the RNA-seq (Appendix Fig S2A) and proteomics (Appendix Fig S2B) workflow. Similar to transcriptomics, a large number of neuronal proteins displayed preferential localization to either the somatic or process compartment (Fig 1E). For most differentially localized mRNAs, the respective proteins showed a corresponding compartment-specific expression (e.g. Syn1, Shank2/3, Dlg4, Homer1, Polr2c) (Fig 1F). This suggests that local mRNA translation in processes contributes to the compartment-specific expression of those genes. However, for some genes (e.g. the presynaptic Sv2a and Syt1), mRNA and protein enrichment did not match, suggesting that protein transport plays a major role in the subcellular localization of these genes.

Having validated our cell culture model, we went on to investigate dynamic compartment-specific changes in the transcriptome/ proteome upon synaptic downscaling in response to $48 \mathrm{~h}$ PTX application. Using Ribo(-)-RNAseq, we identified hundreds of RNAs that were differentially expressed $(q<0.05)$ between PTX- and mocktreated conditions in the somatic ( $n=972 ; 431$ down/541 up) and process ( $n=949 ; 525$ down/424 up) compartment (Fig 2A). Of those, about $50 \%$ (463) were common to both compartments, demonstrating that a large fraction of PTX-responsive RNAs displays compartment-specific regulation (Fig 2B). To better visualize compartment-specific effects of PTX, we plotted logFC in somata vs $\operatorname{logFC}$ in processes and colour-coded gene groups according to their regulation (Fig 2C). Examples of candidate mRNAs within each of these gene groups are marked in Fig 2C, e.g. process up (Sort1), process down (Srcin1, Add2, Dnajc6) and genes commonly up(Plk2) or downregulated (Camk2a, Atp2b4, Shank2) in either compartment. In addition to protein-coding mRNAs, PTX also altered the expression of many non-coding RNAs in a compartmentspecific manner, e.g. long non-coding RNAs (lncRNAs) and primary microRNAs (miRNAs) (Appendix Fig S3). Furthermore, we detected numerous examples of genes which display compartmentspecific alterations in exon/UTR usage upon PTX treatment (Fig EV3A-D), e.g. BDNF (Fig EV3E). Exon-Intron split analysis (EISA) (Appendix Fig S4) and differential expression analysis based on RNA half-lives (Appendix Fig S5) further indicates that 
A

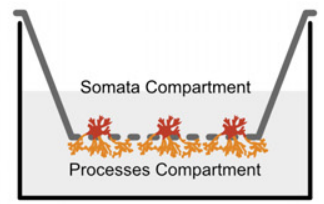

B

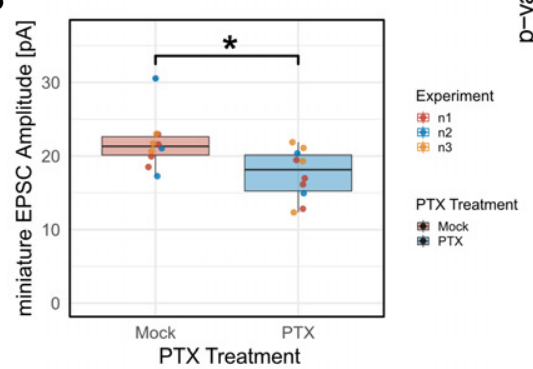

E

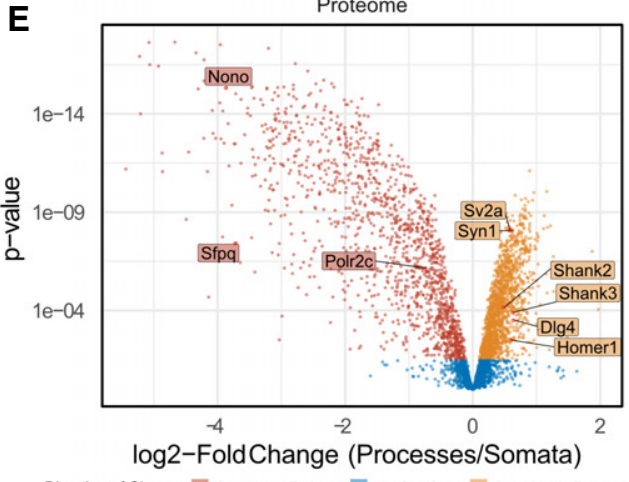

Direction of Change a somatically Eniched a not Signifont a Dentitioly Enichod
C

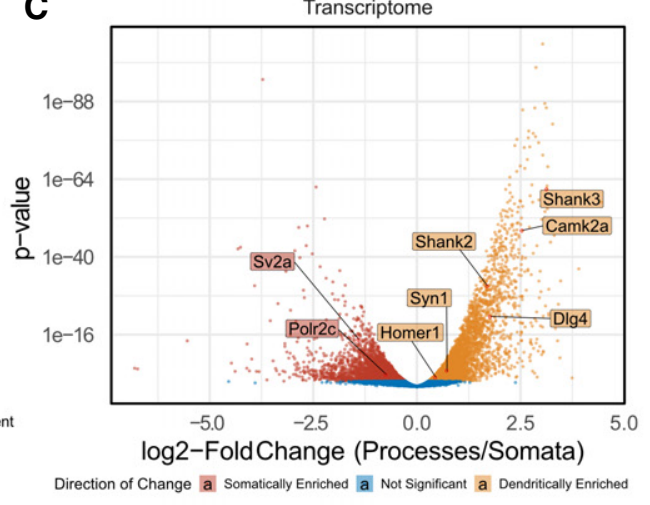

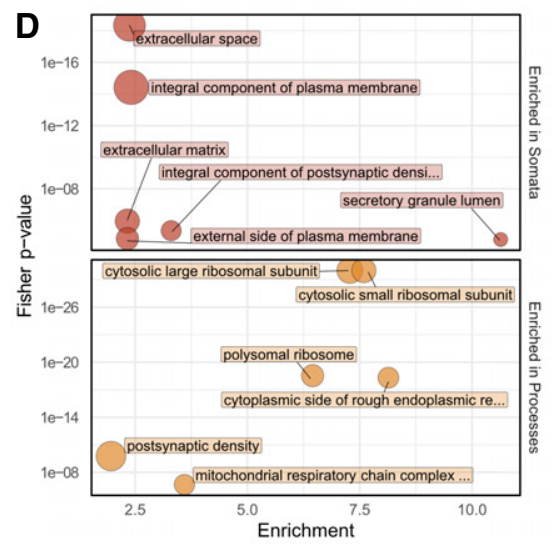

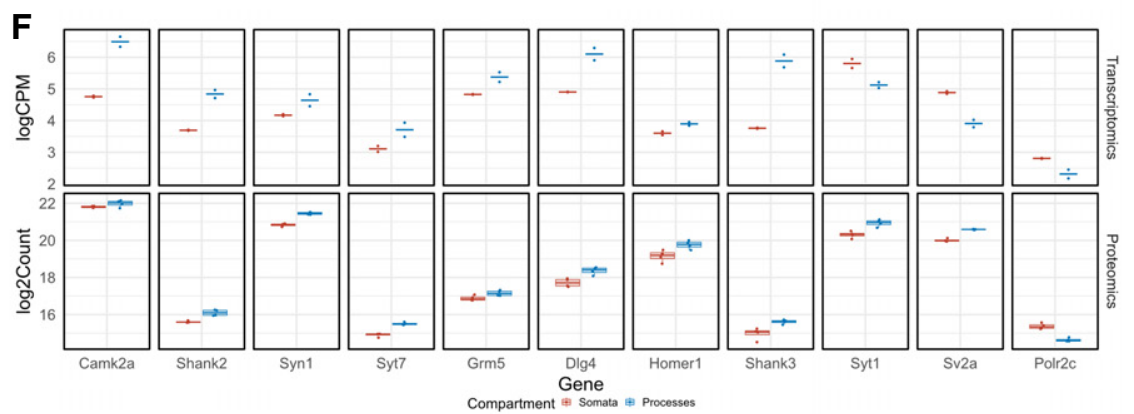

Figure 1. Compartment-specific localization of neuronal transcripts and proteins.

A Schematic of the workflow for the culture of primary hippocampal neurons and $48 \mathrm{~h}$ of PTX treatment.

B Quantification of miniature EPSC amplitudes in mock- and PTX-treated hippocampal neurons. ( $n=10$ neurons per condition from three independent experiments, independent two-sample Wilcoxon-Mann-Whitney U-test, * $P<0.05$ ).

C Volcano plots demonstrating enrichment of transcripts in either the somatic (red) or process (yellow) compartment with representative genes highlighted.

D Top Gene Ontology Pathway Analysis for transcripts enriched in the somatic and process compartment.

E Volcano plots demonstrating enrichment of proteins in either the somatic (red) or process (yellow) compartment with representative genes highlighted.

F Quantification of the highlighted genes enriched in either the somata or processes at the transcript or protein level. (Upper row: Scatterplots of transcript level; $n=2$; crossbar represents mean. Lower row: Boxplot of protein levels; $n=4)$.

Boxplots: central line: median; box: $25^{\text {th }}$ to $75^{\text {th }}$ percentile; whiskers: until last data point within $1.5 \times$ interquartile range (IQR).

Linear model was used to fit transcriptome and proteome data and subsequently contrasted using likelihood ratio testing (transcriptome) or empirical Bayes statistics (proteome) for compartment effects.

PTX-dependent regulation of many genes cannot be solely explained by transcriptional effects. To obtain insight into the biological function of compartment-specific regulation by PTX, we performed GO term analysis (Fig 2D; Appendix Fig S6). Genes specifically upregulated in the process compartment were often associated with the cell cycle (e.g. Cdc20) or metabolism, whereas "somata up genes" were enriched in neuropeptide signalling (e.g. Tac1) and intracellular trafficking (e.g. Sort1). Consistent with PTX-mediated downscaling of excitatory synapses, PTX-downregulated genes in both compartments were highly enriched for regulators of excitatory synaptic transmission. However, whereas the majority of "somata down genes” encode for regulators of synaptic excitation, e.g. ionotropic glutamate receptors (subunits of the AMPA, NMDA and Kainate type) and transmembrane transporters (e.g. several members of the Slc family), "process down genes" mostly encode for structural components of the postsynaptic density, e.g. scaffolding (e.g. Shank1,3, Dlg3, Srcin1, Syngap1, Dlgap1, Homer2/3) and cytoskeletal proteins (e.g. Add2, Dnm1, Map1a/b/s). Taken together, our results demonstrate that compartment-specific regulation of the neuronal transcriptome during synaptic scaling is more pervasive than previously anticipated.

We went on to validate selected high-ranking candidates that display robust compartment-specific regulation by PTX, particularly focusing on genes that are preferentially regulated in the process compartment. Using qPCR, we validated PTX-dependent differential regulation of several genes, such as Sort1, Add2, Shank2 and Srcin1 (Fig 3A, Appendix Fig S7). Furthermore, we detected up-regulation of Plk2 in either compartment, consistent with a previous report (Seeburg et al, 2008). Compartment-specific regulation by PTX was further confirmed for Add2, Sort1 and Dnajc6 at the single neuronal 

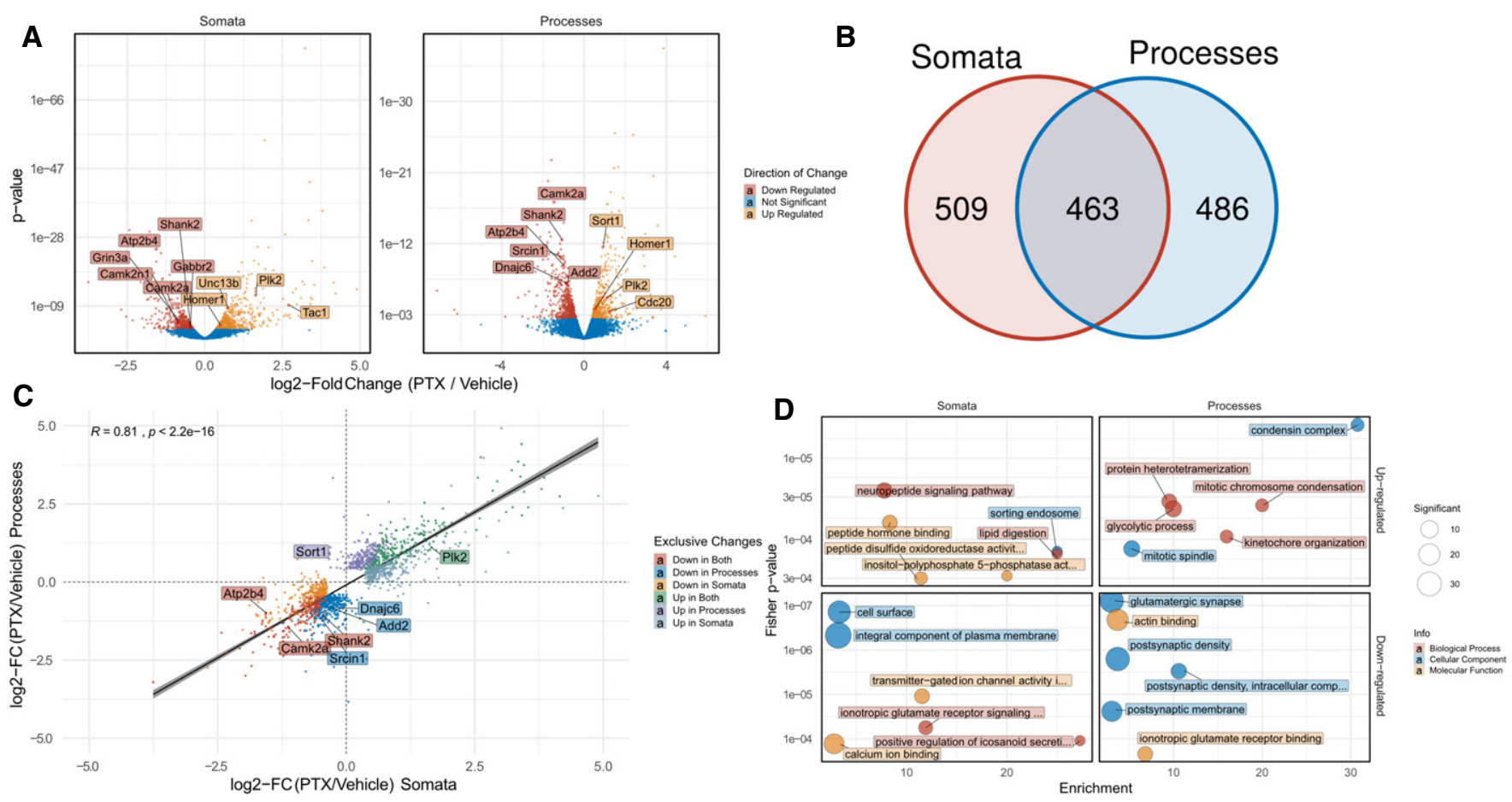

Figure 2. Compartment-specific regulation of neuronal transcripts by PTX.

A Volcano plots representing transcript down- (red) or up-regulation (yellow) after $48 \mathrm{~h}$ PTX in the somatic and process compartment. Representative transcripts are highlighted and labelled. Linear model was used to fit transcriptome data and subsequently contrasted using likelihood ratio testing for PTX treatment effects across compartments.

B Venn-Diagram of differentially expressed transcripts between somatic and process compartment

C Spearman rank correlation between $\log _{2}$-fold changes of differentially expressed genes significant in either the somatic or process compartment colour-coded for exclusive changes in the respective compartments. Representative genes are highlighted and labelled. The statistical significance of Spearman correlations was calculated in the standard way, using Spearman's rho statistic. Regression lines indicate fitted linear models, with the light grey shaded areas depicting the $95 \%$ confidence interval.

D Top 6 Gene Ontology Pathways enriched in differentially expressed genes in either the somatic or process compartment colour-coded for Cellular Component (blue), Molecular Function (yellow) or Biological Process (red).

level using single-molecule fluorescent in situ hybridization (smFISH) (Fig 3B and C; Appendix Fig S7). smFISH further allowed us to distinguish between dendritic and axonal processes. Whereas PTX selectively reduced dendritic Add2 and Dnajc6 mRNA puncta, Sort1 mRNA puncta were significantly increased in dendrites. For all three genes, levels of somatic mRNA puncta were unaffected by PTX treatment.

We next wondered whether the PTX-induced compartmentspecific changes in transcript levels translated into corresponding changes in protein. Although dynamic time- and polarity-dependent changes in the neuronal proteome have been previously demonstrated in synaptic scaling using labelled proteomics (Schanzenbacher et al, 2016; Schanzenbacher et al, 2018; Dorrbaum et al, 2020), these studies did not address potential compartment-specific effects. Therefore, we extracted proteins from PTX-treated compartmentalized hippocampal neuron cultures and quantified proteomewide changes using label-free proteomics. In total, we detected peptides corresponding to 4,250 different proteins with our approach. Differential expression analysis revealed that 660 proteins were significantly changing in somata and 320 proteins in processes, respectively (FDR $<0.5$, Fig 4A; Appendix Fig S8). Much like our observations taken from RNA-seq, we detected substantial compartment-specific regulation of protein levels during synaptic downscaling, with only 162 proteins commonly regulated in both the somatic and process compartment (Fig 4B). Comparable with the RNA-data, GO-term analysis of differentially expressed proteins indicated a strong enrichment of synaptic proteins among PTXdownregulated proteins (Appendix Fig S9). Importantly, although many of the top-ranking PTX-regulated mRNAs were not detectable by proteomics, PTX-dependent changes in RNA and protein levels in both compartments strongly correlated, in particularly for genes encoding synaptic proteins (Fig 4C and EV4A and B; Appendix Fig S10A and B), e.g. preferential downregulation of Shank1, Dlg3, Crtc1 and Map1a in the process- and Grin2b, Slc2a13 in the somatic compartment, respectively. A correlation of PTX-dependent changes in RNA and protein levels is also observed for most of the candidates validated by qPCR (Fig 4D). However, for a few genes, PTXdependent changes in protein and RNA levels did not correlate. For example, Sort1 is significantly upregulated at the RNA level (Fig 3C), but decreased at the protein level in the process compartment (Fig 4D). This result suggests that additional regulatory mechanisms at the level of mRNA translation and/or protein turnover 


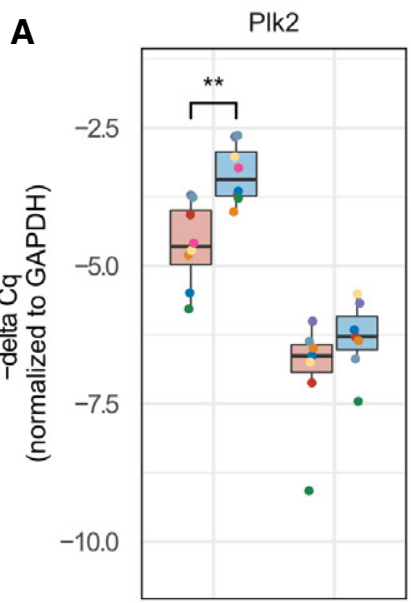

Somata Processes

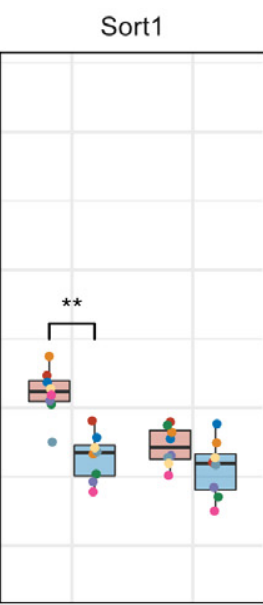

Somata Processes

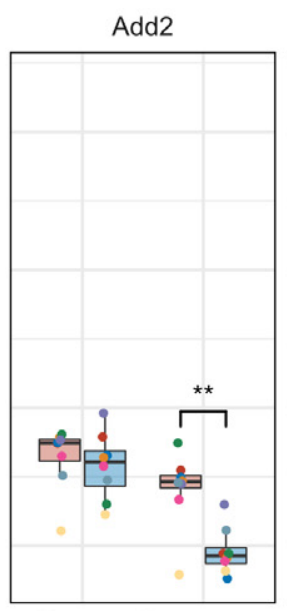

Somata Processes Compartment

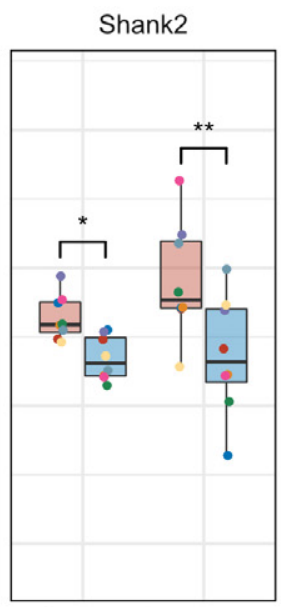

Somata Processes

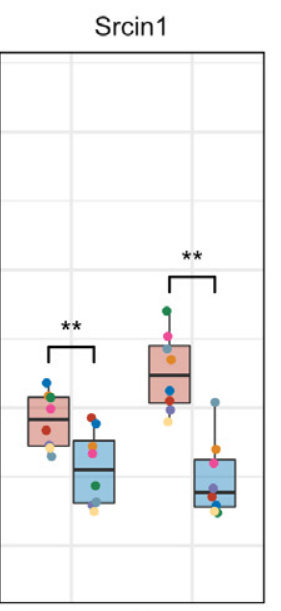

Somata Processes
Experiment

宛1

宛2

亩 $n 3$

审 4

审 n5

审 n6

审 $n 8$

PTXTreatment

审 Mock

审 PTX
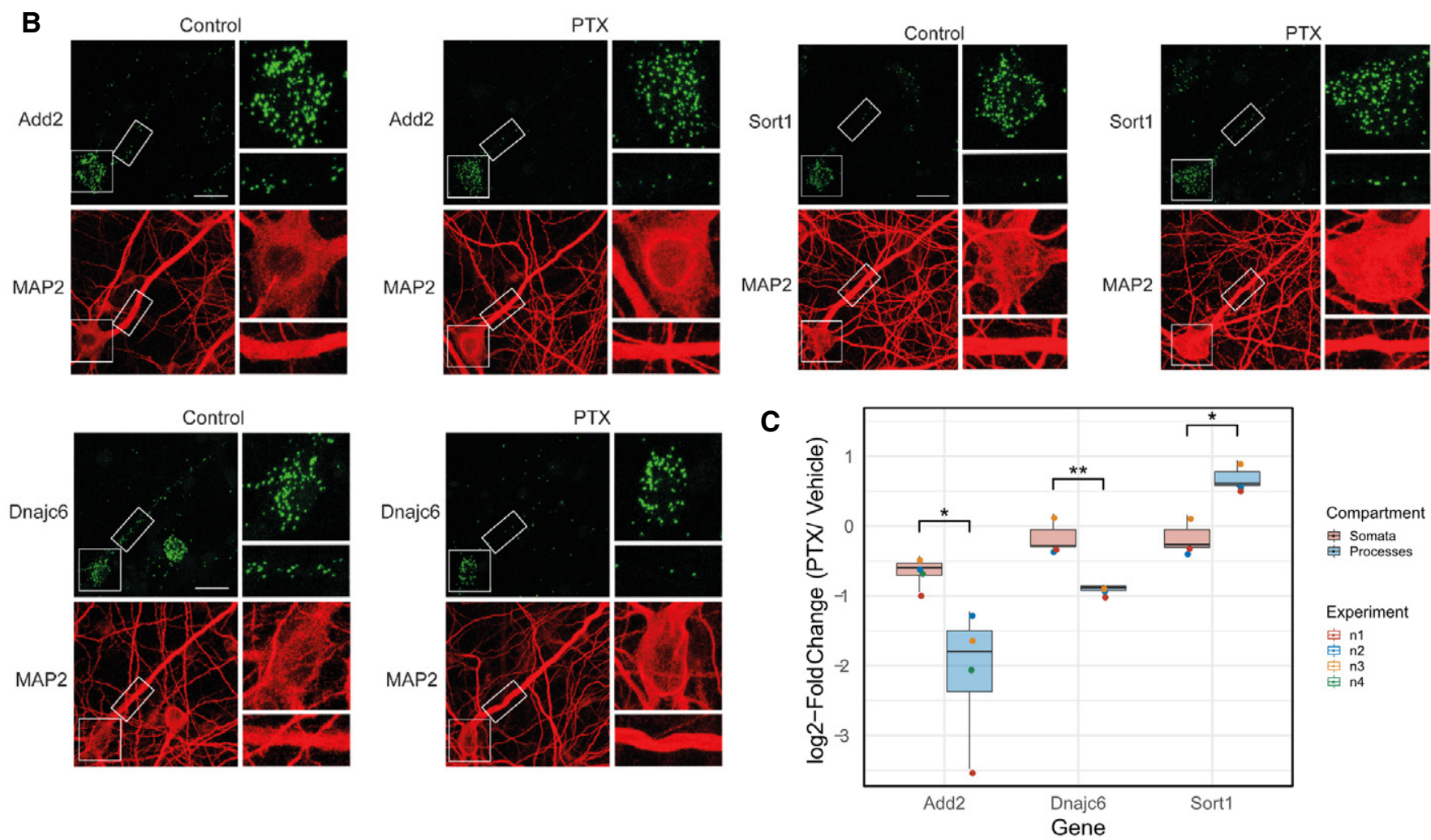

Compartmen

审 Somata

Experiment

穴 $\mathrm{n} 1$

审 $\mathrm{n} 2$

审 $n 4$

\section{Figure 3. Validation of compartment-specific regulations.}

A Real-time quantitative PCR (RT-qPCR) of transcripts changing differentially between compartments using the compartmentalized cultures after 48 h PTX treatment. $n=8$ independent biological replicates (except for Plk2 $(n=7)$, where one measurement couldn't be performed due to insufficient cDNA); PTX effect was assessed by three-way ANOVA followed by Tukey's post hoc multiple comparison test; ${ }^{*} P<0.05 ;{ }^{* \star} P<0.01$.

B Representative images of single-molecule FISH (smFISH) in either control or 48 h PTX-treated rat hippocampal neurons (DIV20) using probes specific for Add2, Dnajc6 and Sort1 (green). MAP2 immunostaining (red) was used to visualize neuronal somata and dendrites. Inserts at higher magnification illustrate PTX-dependent changes in dendritic RNA puncta. Scale bar $=10 \mu \mathrm{m}$

C Quantification of (B). ( $n=3-4$ independent biological replicates with 8-10 cells averaged per condition and replicate; two-sample Student's $t$-test; ${ }^{\star} P<0.05$; $\left.{ }^{\star *} p<0.01\right)$.

Data information: Boxplots: central line: median; box: $25^{\text {th }}$ to $75^{\text {th }}$ percentile; whiskers: until last data point within $1.5 \times$ interquartile range (IQR). 
contribute to PTX-dependent process expression of those genes. To obtain further insight about compartment-specific effects of PTX on protein expression, we compared our dataset to a previous publication which investigated protein synthesis and degradation in response to the GABA-A-R antagonist bicuculline independent of cellular compartments (Dorrbaum et al, 2020). In general, we observed a significant positive correlation between protein logFC, suggesting that PTX and Bic induce similar proteomic changes (Fig EV5A). Interestingly, when focusing only on nascent proteins (those which are arguably more strongly correlated with RNA abundance), the correlation was much stronger for proteins which significantly change in the process compared to the somatic compartment (Fig EV5B-E). Accordingly, many of the proteins we found strongly downregulated in the process compartment (e.g Khsrp, Ccar) were only modestly altered in the study by Dorrbaum et al. Together, this result underscores the utility of our compartment-specific approach for the detection of local changes in protein abundance at high spatiotemporal resolution.

In an effort to unequivocally address local regulation of nascent protein synthesis, we performed the previously published puromycin proximity ligation assay (PLA) (tom Dieck et al, 2015) for selected candidates (Fig 5). For this analysis, we chose Camk2a (downregulated in both somatic and process compartment) and Syn1 (downregulated selectively in the process compartment) based on our results from RNA-seq and proteomics. Whereas puncta labelling nascent Camk2a protein were significantly reduced in both the somata and dendrites of PTX-compared to mock-treated neurons (Fig 5A and C), Syn1 puncta density was only significantly reduced in dendrites and remained unchanged in somata (Fig 5B and C). In the absence of puromycin treatment (lower panels in Figs 5A, B and D), signal intensity was strongly reduced, demonstrating the specificity of the assay. Together, these data confirm the compartmentspecific regulation of nascent protein synthesis by PTX for selected candidates.

Next, we sought to characterize the molecular mechanisms which underlie the compartment-specific regulation by PTX, focusing on dynamic changes in mRNA levels. Untranslated regions (UTRs) of mRNAs are considered as important determinants of both mRNA localization and post-transcriptional regulation. $3^{\prime}$ UTRs in particular contain sequence elements for trans-acting posttranscriptional regulators, such as RNA-binding proteins (RBPs) and miRNAs (Fig 6A). We first determined the average $3^{\prime} U T R$ length in genes regulated in a compartment-specific manner (Fig 6B). 3'UTRs of PTX-downregulated genes were slightly longer in comparison with upregulated genes in both the somatic and process compartment, although the difference was only statistically significant in the former compartment. Thus, $3^{\prime}$ UTR length differences alone unlikely explain the high degree of compartment-specific gene regulation during synaptic scaling.

Subsequently, we investigated the compartment-specific enrichment of specific sequence motifs located within $3^{\prime}$ UTRs. We first interrogated overrepresentation of RBP binding motifs determined in a previous large-scale in vitro study (Ray et al, 2013) in the $3^{\prime}$ UTRs of PTX-regulated mRNAs (Fig 6C). Whereas only five motifs were significantly (adjusted $P$-value $<0.05$ ) overrepresented in PTX-regulated somatic mRNAs, 16 motifs fulfilled these criteria in PTX-regulated process-enriched mRNAs (Fig 6C). Interestingly, only one motif in total could be recovered from PTX-upregulated genes, suggesting that RBPs acting by $3^{\prime}$ UTR-dependent mechanisms are mostly involved in PTX-mediated gene repression. Among the motifs, most highly enriched in PTX-downregulated genes in somata is the Rbfox 1 consensus binding site (GCAUG). This fits well with our previous results showing an important role of Rbfox1 in synaptic scaling at the level of the entire neuron (Rajman et al, 2017). Concerning process-enriched RBP motifs, many of them are recognized by known regulators of mRNA stability and translation (e.g. hnRNP-K (Folci et al, 2014; Bottini et al, 2017; Leal et al, 2017), Nono (Bottini et al, 2017) and Lin-28 (Amen et al, 2017)). This suggests an important contribution of RBPs to compartment-specific gene regulation during synaptic scaling.

miRNAs represent an additional class of gene-specific regulatory molecules that negatively affect mRNA expression by recruiting a silencing protein complex to specific sites (miRNA-binding sites, MBS) in $3^{\prime}$ UTRs (Bartel, 2009). To estimate miRNA contribution to PTX-dependent regulation, we performed an MBS enrichment analysis. Briefly, the number of MBS within a gene set of interest (i.e. PTX-downregulated genes in the process compartment) were compared to those present in the sequencing background (Fig 6D; see Materials and Methods for further details). We calculated enrichment scores for MBS of neuronally expressed miRNAs $(n=221)$ in the $3^{\prime}$ UTR of genes that are downregulated by PTX in a compartment-specific manner. In both compartments, MBS were on average more abundant in PTX down- compared to upregulated genes $\left(\log _{2}[\mathrm{PTX} /\right.$ control $]>0$; Fig 6E), as expected. However, the enrichment value ( $\log _{2}$-fold ratio) obtained from processes was significantly higher compared to somata (Fig 5E; $P<0.001$ ). Taken together, these results suggest that miRNA-dependent mRNA repression plays a particularly important role in the process compartment during synaptic downscaling. At the level of individual miRNA families, specific sets of MBS were significantly enriched over background in PTX-downregulated genes in either the somatic or process compartment (Fig 6F). Our approach independently identified miR129-5p, which we previously showed to be required for synaptic downscaling (Rajman et al, 2017), as the miRNA whose binding motifs were most strongly enriched in PTX-downregulated genes in the somatic compartment. This finding indicates that our approach is able to identify functionally important miRNAs solely on the basis of differential expression analysis. On the other hand, our enrichment analysis identified an overrepresentation of several miRNAbinding motifs in mRNAs that are specifically downregulated by PTX in the process compartment, such as the activity-regulated miR132/212 (Wayman et al, 2008), miR-24-3p, miR-125a-5p, and the miR-379-410 cluster members miR-329-3p and miR-485-5p (Lackinger et al, 2019). One miRNA that appears to be specifically active in dendrites during PTX-induced downscaling is miR-34c-5p, a member of the miR-34/449 family (Fig 5F, Appendix Fig S11). A total of 53 PTX-downregulated genes in processes contain miR-34/ 449 binding motifs in their $3^{\prime}$ UTRs, including important synaptic regulatory proteins such as Add2, Shank3, Cntn2, Cacng2, Scn2b, Mpp2 and Crtc1 (Torc1) (Fig 7).

Taken together, compartment-specific regulation of neuronal transcripts during synaptic downscaling is associated with specific $3^{\prime}$ UTR features, including unique miRNA and RBP motifs. Posttranscriptional regulation by miRNAs and RBPs appears to be particularly widespread during PTX-mediated downregulation of synaptic genes in the process compartment. 
A
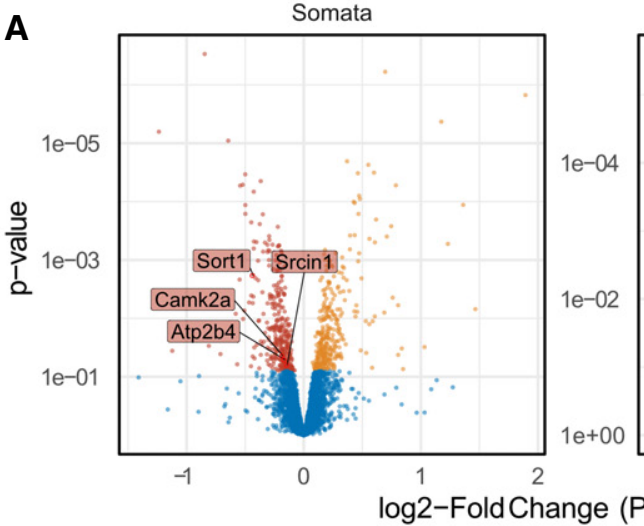

Processes

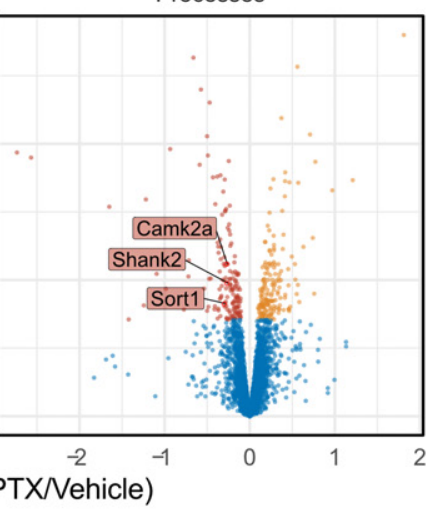

B

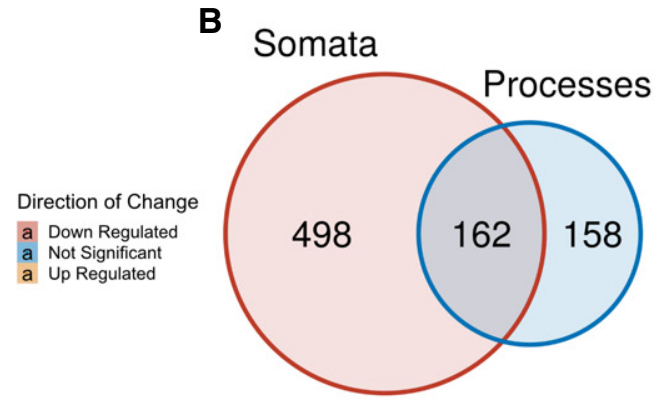

C

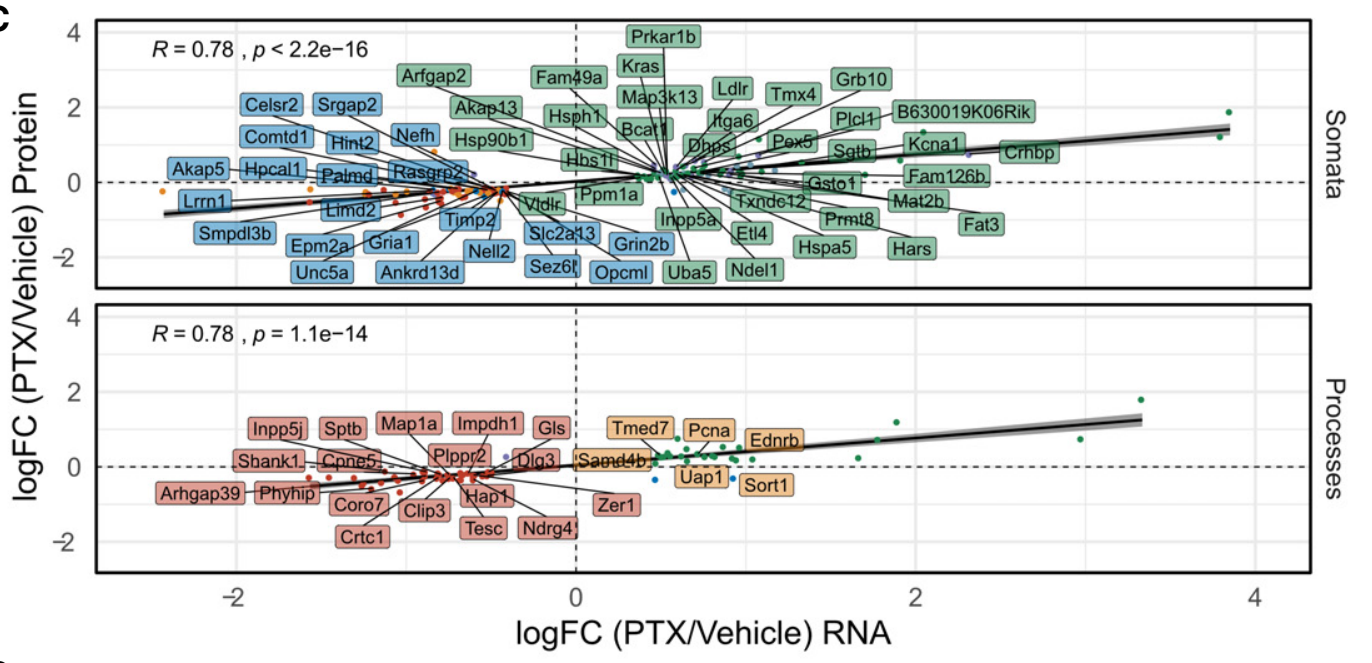

Exclusive Changes (Protein vs RNA)

- Down in Both

- Down in Proteins

- Down in RNA

- Up in Both

- Up in Proteins

- Up in RNA

Exclusive Changes

(Processes vs Somata)

a Down in Processes

a Down in Somata

Up in Processes

a Up in Somata
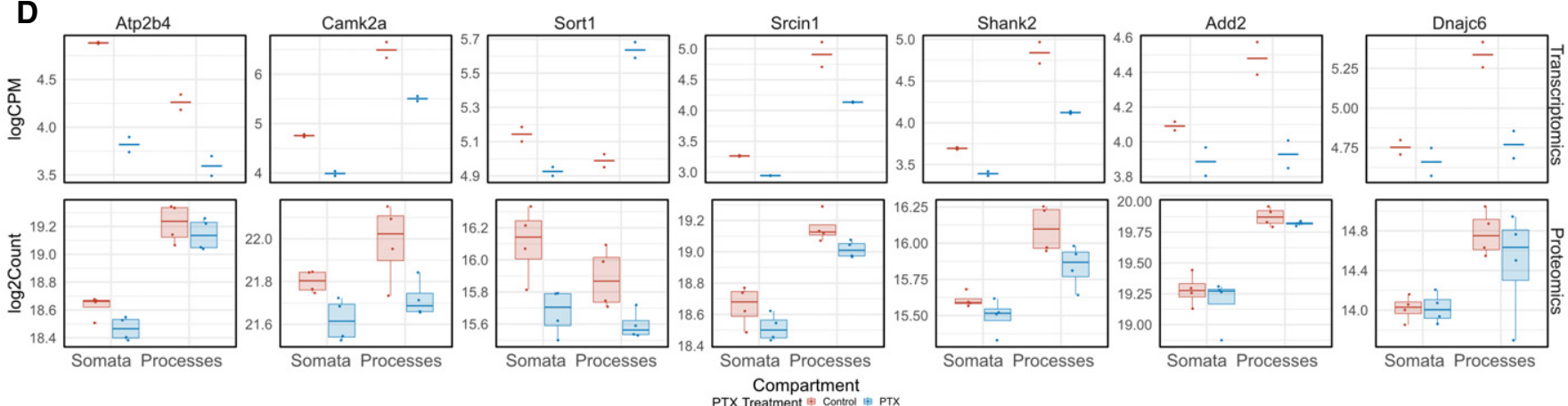

\section{Figure 4. Compartment-specific regulation of neuronal proteins by PTX.}

A Volcano plots representing proteins down- (red) or up-regulation (yellow) after $48 \mathrm{~h}$ PTX in the somatic and process compartment. FDR $<0.5$. Representative proteins are highlighted and labelled. Linear model was used to fit proteome data and subsequently contrasted using empirical Bayes statistics for PTX treatment effects across compartments.

B Venn-Diagram of differentially expressed proteins between somatic and process compartment.

C Spearman rank correlation between $\log _{2}$-fold changes of differentially expressed genes changing at the transcript and protein level. The statistical significance of Spearman correlations was calculated in the standard way, using Spearman's rho statistic. Regression lines indicate fitted linear models, with the light grey shaded areas depicting the $95 \%$ confidence interval.

D Representative plots of genes changing after PTX at the transcript and protein level. (Upper row: Scatterplots of transcript level; $n=2$; crossbar represents mean. Lower row: Boxplot of protein levels; $n=4)$. Boxplots: central line: median; box: $25^{\text {th }}$ to $75^{\text {th }}$ percentile; whiskers: until last data point within $1.5 \times$ interquartile range (IQR).

\section{Discussion}

In this study, we provided the first comprehensive analysis of compartmentalized gene expression during synaptic plasticity, more specifically in response to homeostatic synaptic downscaling induced by prolonged treatment of primary rat hippocampal neurons with the GABA-A-R blocker PTX. A major outcome of our study was the observation that PTX treatment induces 
A

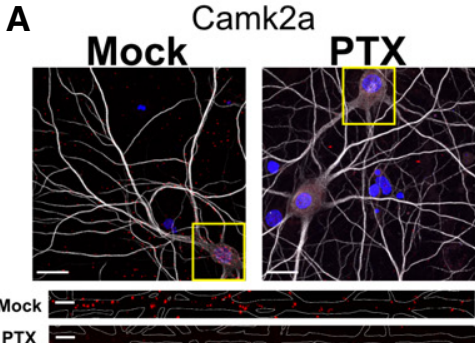

PTX
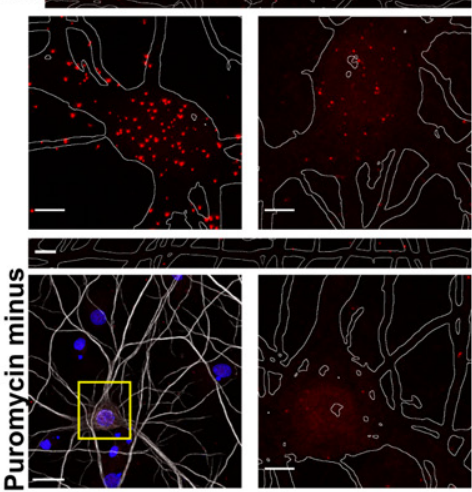

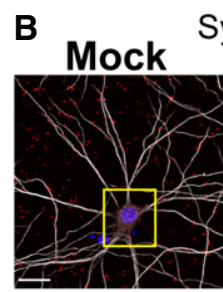

Mock -
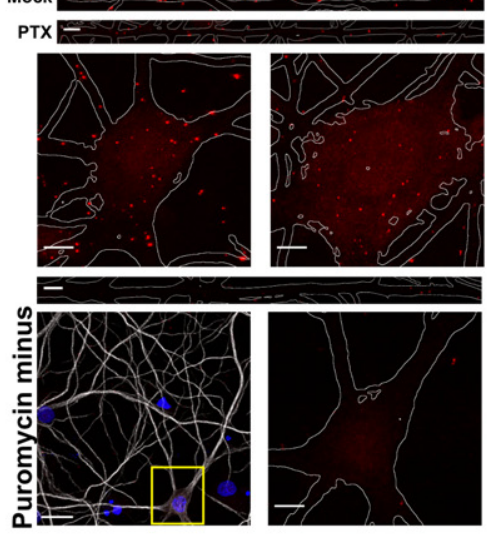

C

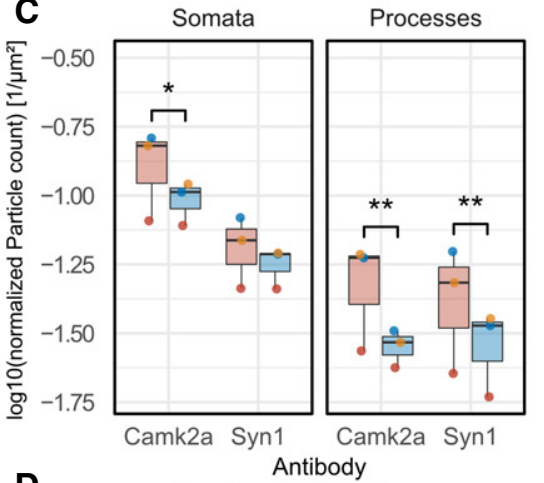

Experiment

贯 $n 1$

亩 $n 3$

PTX-Treatment

Mock

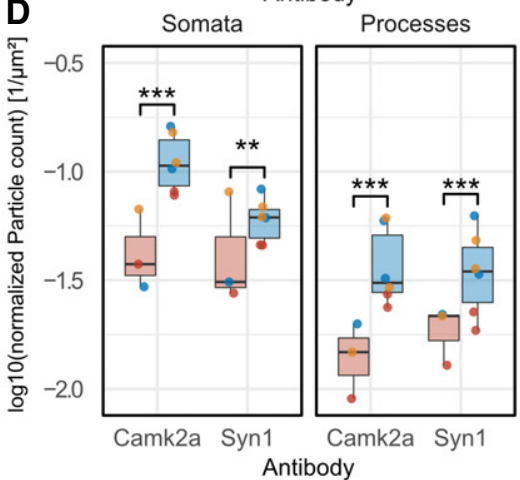

Experiment

戛 $n 1$

审 $n 2$

Puromycin Treatment Puro-Minus Puro-Plus

Figure 5. PTX-mediated regulation of local translation.

A Representative pictures of nascent Camk2a-peptides in mock- and PTX-treated neurons. Upper Panels: Representative overview images with merged channels (grey: MAP2-staining, red: Duolink-PLA signal, blue: Hoechst nuclear staining). Middle Panels: Magnifications of boxed areas marked in (A). MAP2 signal was used to outline somata and dendritic processes (white lines). Lower Panels: Negative control of puromycin-untreated cells with representative Duolink signal in dendrite and soma, respectively. (Scale bars: in overview images $20 \mu \mathrm{m}$, in inserts of higher magnification $5 \mu \mathrm{m}$ ).

B Same as (A) with nascent Synapsin1-peptides.

C Quantification of Duolink-particle density of (A) and (B) assessing PTX treatment effect. $n=3$ independent experiments with 10 images averaged per condition and experiment; Three-way ANOVA followed by Tukey's post hoc multiple comparison test; ${ }^{\star} P<0.05 ;{ }^{* \star} P<0.01$ ).

D Quantification of Duolink-particle density of (A) and (B) assessing Puromycin-treatment effect. $n=3$ independent experiments for Puromycin-Minus and $n=6$ data points for Puromycin-Plus conditions (PTX and mock conditions combined together across $n=3$ independent experiments, taken into account in ANOVA model) with 10 images averaged per condition and experiment; three-way ANOVA followed by Tukey's post hoc multiple comparison test; ${ }^{\star \star} P<0.01$, $\left.{ }^{\star \star \star} P<0.001\right)$.

Data information: Boxplots: central line: median; box: $25^{\text {th }}$ to $75^{\text {th }}$ percentile; whiskers: until last data point within $1.5 \times$ interquartile range (IQR).

compartment-specific changes in the neuronal transcriptome and proteome. The existence of local and global mechanisms in homeostatic plasticity has been known for some time (Turrigiano, 2012), but a clear picture of how homeostatic feedback is structured at the molecular level has not yet emerged. Our study lends further support to the hypothesis that local synaptic and neuron-wide mechanisms co-exist in neurons and act in concert to counteract excessive excitation which would inevitably occur if feedforward Hebbian plasticity operated unconstrained (Rabinowitch \& Segev, 2008).

What distinguishes the somatic and process compartment during synaptic scaling? Some clues might come from our GO-term analysis, which showed a strong overrepresentation of genes involved in the regulation of neuronal excitability (e.g. ion channels, transporters, receptors) in the cell body, whereas the majority of regulation of structural synaptic genes (e.g. postsynaptic scaffolds, cytoskeletal proteins) occurred in the process compartment (Fig 7). Consistent with this observation, many of the process-regulated mRNAs are known dendritic mRNAs, e.g. Shank1/3 and Homer2 and Syngap1, and have recently been shown to be preferentially translated in the synaptic neuropil in vivo (preprint: Glock et al, 2020). On the other hand, "neuronal excitability genes" encode mostly transmembrane proteins, for which the somatic biosynthesis machinery might represent the major source, although dendritic translation has been demonstrated for some of these genes. Thus, mRNA sorting under basal conditions might already pre-determine compartment-specific responsiveness during scaling or other plasticity-inducing cues.

What are the molecular mechanisms underlying the compartment-specific regulation during synaptic scaling? In principle, compartment-specific mRNA changes could be a result of altered mRNA transcription, transport, stability and translation, or combinations thereof. Our experimental setup does not allow to disentangle these intimately coupled processes. However, several lines of evidence suggest that increased mRNA degradation could play a major role, at least in the context of downregulation of synaptic gene expression in processes. First, mRNA and protein levels of differentially expressed genes strongly correlated in both 
A

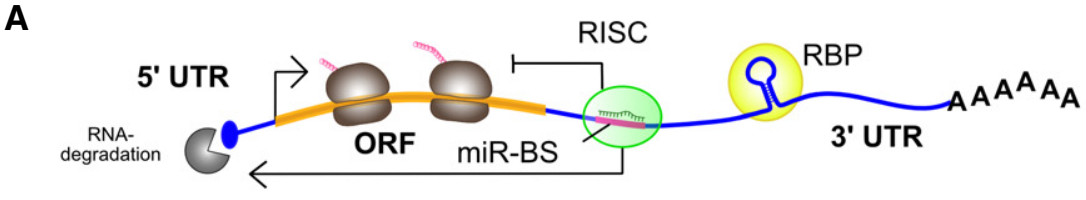

B
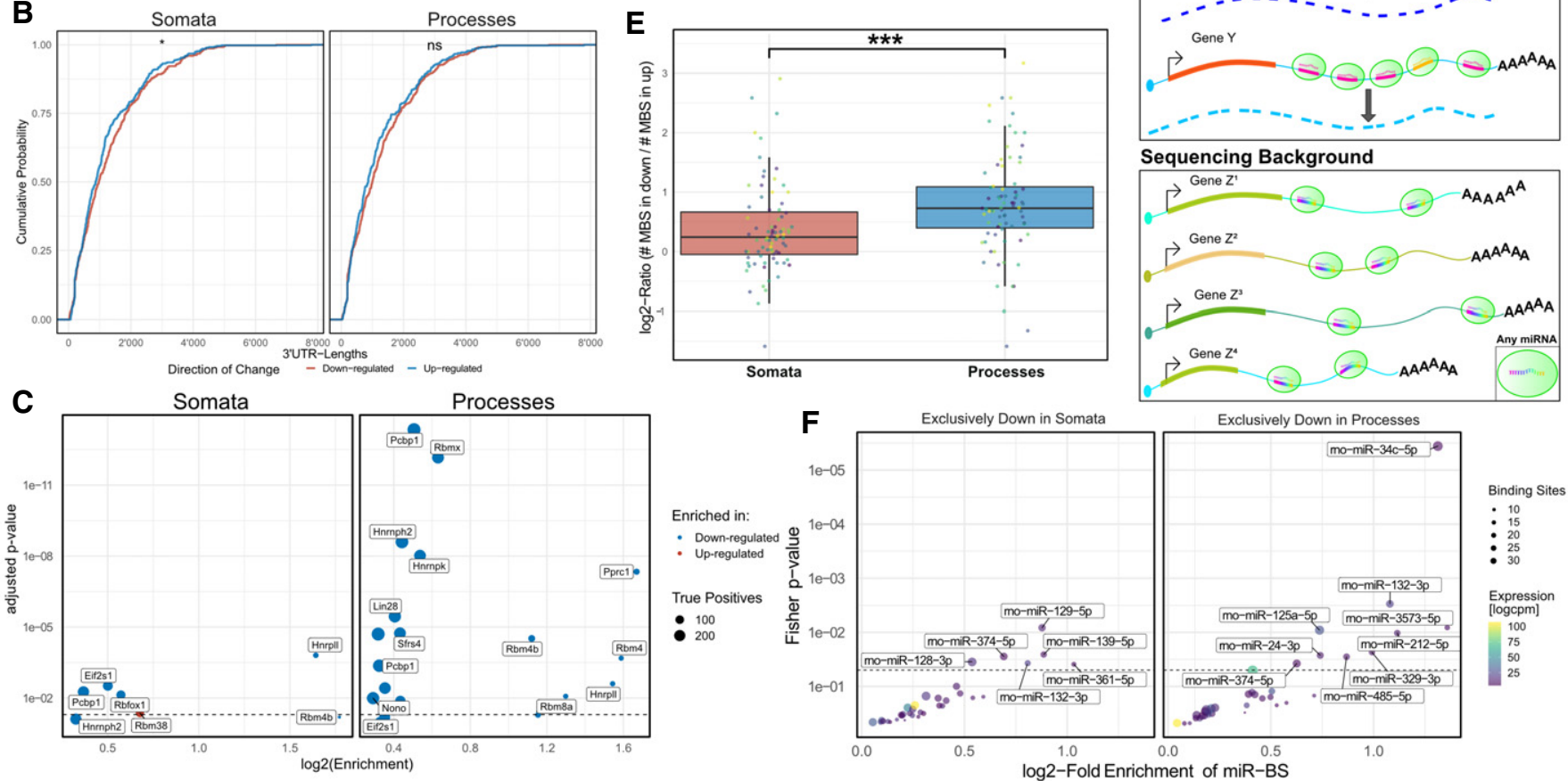

Figure 6. $3^{\prime}$ UTR-dependent mechanisms of regulation by RBPs and miRNAs.

A Schematic of cis-acting RNA-binding proteins (RBPs) and microRNAs (miRNAs) and their effects on mRNA stability.

B Cumulative distribution of $3^{\prime}$-UTR lengths of down- and upregulated genes in either compartment. Two-sample Kolmogorov-Smirnov test; ns $>0.05$, ${ }^{*} P$-value $<0.05$.

C Enrichment analysis for RBP motifs in down- and upregulated genes in either compartment. Fisher's exact test was used to perform enrichment analysis; FDRcorrected.

D Schematic of miRNA-binding site (MBS) enrichment. PTX-mediated synaptic downscaling might lead to a local increase in miRNA abundance or activity. These effects can be potentially captured by testing for an enrichment of MBS in $3^{\prime}$ UTRs of downregulated genes in comparison with the sequencing background (expressed genes).

E $\log _{2}$-Ratios of the number of miRNA-binding sites found in significantly up- vs downregulated genes in the somata (red) or processes (blue). Elevated ratio in processes indicates that miRNA-binding sites are more frequent in transcripts downregulated by PTX in processes. Non-parametric Mann-Whitney $U$-test was used to test difference (mean somata: 0.37 vs mean processes: 0.783 ); ${ }^{\star \star \star} P$-value $<0.0001 ; n=80$ miRNAs, represented as coloured data points. Boxplots: central line: median; box: $25^{\text {th }}$ to $75^{\text {th }}$ percentile; whiskers: until last data point within $1.5 \times$ interquartile range (IQR).

$\mathrm{F}$ Enrichment of MBS in genes that are exclusively downregulated in somata or processes compared to the sequencing background. The significant miRNA families $\left(P\right.$-value $<0.05$ and $\log _{2}$-fold enrichment $\left.>0.5\right)$ are labelled by a representative miRNA. See Methods for detailed statistical analysis.

compartments (Figs 4C and EV4), which argues in favour of a mechanism which involves alterations in mRNA abundance. Second, PTX did not uniformly reduce mRNA levels of synaptic genes across departments and also did not affect short-lived mRNAs more strongly (Appendix Fig S5), as would have been expected if these changes were purely a result of transcriptional inhibition. Third, we did not obtain evidence for a massive PTX-dependent relocation of candidate mRNAs between compartments from RNAseq and smFISH (Fig 3B), arguing against a major contribution of PTX-regulated RNA transport. In this regard, the inhibitory effects of PTX observed in the local mRNA translation assay (Puro-PLA) might be primarily a consequence of reduced levels of the corresponding mRNAs, although additional effects on mRNA translation efficiency cannot be ruled out. In the future, more direct approaches to investigate local mRNA degradation (e.g. TREAT (Horvathova et al, 2017), 4-thiouridine metabolic labelling (Herzog et al, 2017)) will have to be performed.

The striking overrepresentation of motifs for miRNAs and RBPs in $3^{\prime}$ UTRs of PTX-regulated genes which are selectively downregulated in processes (Fig 6C and F) further suggests that gene-specific post-transcriptional mechanisms might play a particularly important role in the local control of synaptic gene expression. Regarding miRNAs, many of the PTX-downregulated synaptic genes (e.g. Add2, Shank3, Cntn2, Cacng2, Scn2b, Mpp2) contain binding sites for the miR-34/449 family. A synaptic function of miR-34 was very recently reported in Drosophila, where it controls pre- and postsynaptic function at the neuromuscular junction (NMJ) (McNeill et al, 2020). miR-34 has also been implicated in stress-associated 


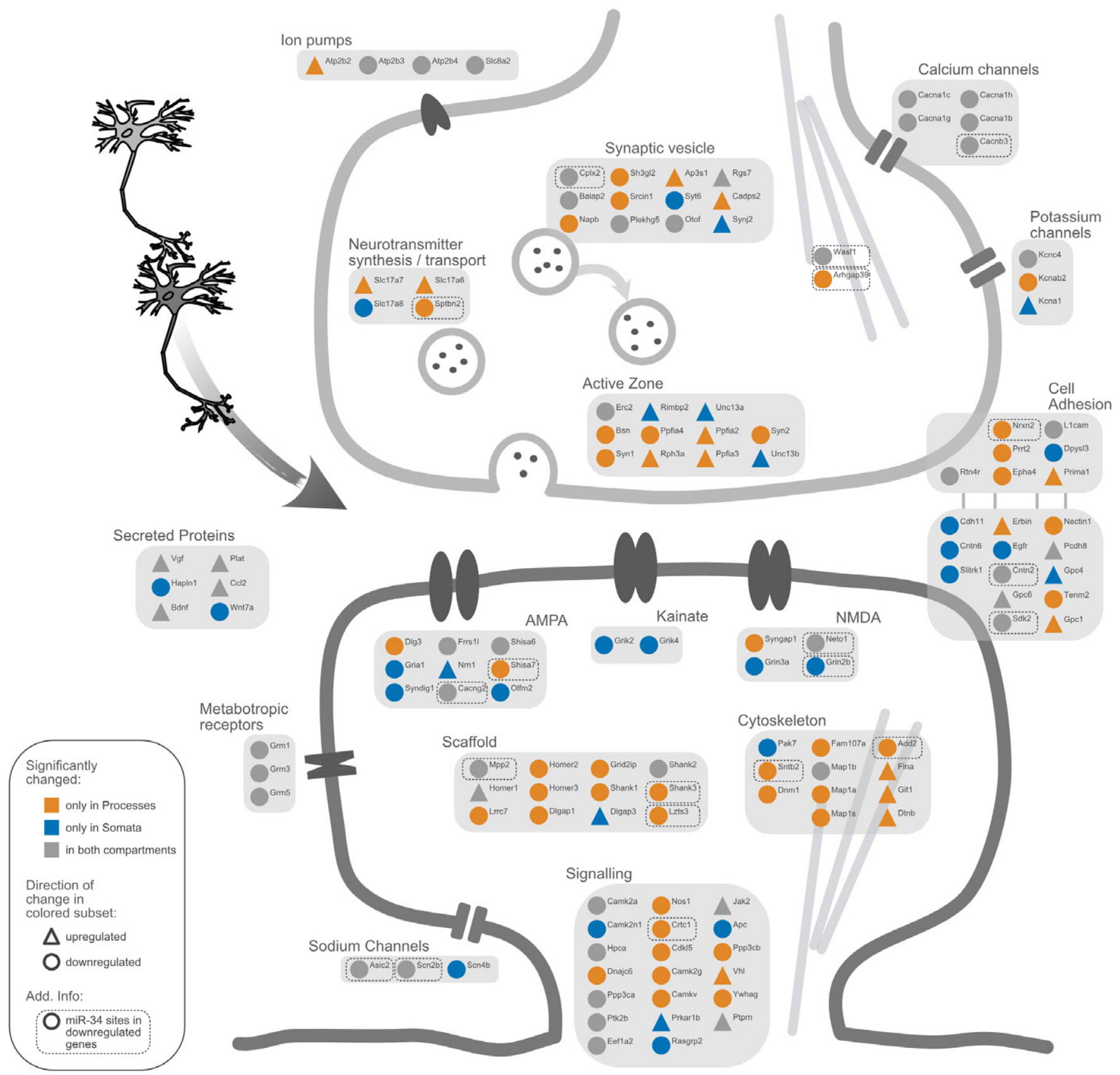

\section{Figure 7. PTX-mediated changes in synaptic genes.}

Schematic representation of significantly changing genes (compartment RNA-sequencing) associated with excitatory synapses. Genes differentially expressed in only one compartment are highlighted with either yellow (processes compartment) or blue (somata compartment). The direction of change is illustrated as symbol shape (circle $=$ sign. downregulated genes, triangle $=$ sign. upregulated genes). miR-34 family binding sites in sign. Downregulated genes are marked with a dashed line.

disorders, e.g. anxiety and epilepsy (Sano et al, 2012), providing potential links between miR-34, synaptic scaling and neurological disease which should be explored in the future. Additional candidate miRNAs for follow-up studies include miR-24, miR-132 and miR-125, which are high-ranking synaptic miRNAs according to a recent study (Epple et al, 2021). Concerning RBPs, motifs for several members of the hnRNP family, Lin-28 and Nono were specifically enriched in "process down genes", consistent with their reported presence in dendritic RNA granules (Kanai et al, 2004). Among them, hnRNP-K might be a strong candidate for follow-up experiments. Since hnRNP-K has mostly stabilizing functions on mRNA (Nagano et al, 2006; Shanmugam et al, 2008; Proepper et al, 2011), one possible scenario is that it counteracts miRNA activity under basal conditions and is subsequently inactivated upon scaling. In a broader context, specific combinations of motifs for trans-acting regulatory factors ("regulons") might underlie the spatiotemporal 
control of neuronal gene expression in response to extracellular cues.

What could be the biological relevance of compartmentalized gene expression during homeostatic scaling? One can speculate that it might allow neurons to maintain excitability while taking into account individual plasticity needs of synapses or dendritic domains (Rabinowitch \& Segev, 2006). Intriguingly, a very recent study described the local allocation of proteins in so-called "synaptic neighborhoods”, about $10 \mu \mathrm{m}$ long dendritic domains, in a related form of homeostatic plasticity, synaptic upscaling (preprint: Chao Sun, 2020). In agreement with our results, many of these allocated proteins might arise from local dendritic mRNA translation. Thus, local or quasi-local mechanisms operating at the level of synapses, neighbourhoods or dendritic domains might represent a common feature of homeostatic plasticity in excitatory neurons.

In conclusion, system-wide approaches like the one presented here might help to disentangle the spatiotemporal logic of the complex regulatory networks underlying synaptic plasticity and homeostasis and their aberrations in diseases such as epilepsy and autism.

\section{Materials and Methods}

\section{Cell culture, transfection and stimulation}

Primary cultures of Sprague Dawley rats (Charles River Laboratories, Sulzfeld, Germany) embryonic hippocampal neurons were prepared as described previously (Schratt et al, 2006) and plated onto porous membrane cell culture inserts as described previously (Bicker et al, 2013). For stimulation, 18DIV neurons were treated either with Picrotoxin (PTX; $100 \mu \mathrm{M}$ final concentration, Sigma) or vehicle (ethanol absolute) for $48 \mathrm{~h}$ and lysed at 20DIV. All animal experiments were carried out under institutional guidelines (ZH196/ 17 Kanton Zürich Gesundheitsdirektion Veterinäramt).

\section{Electrophysiology}

Whole cell patch clamp recordings were performed on an upright microscope (Olympus BX51WI) at room temperature. Data were collected with an Axon MultiClamp 700B amplifier and an Digidata 1550B digitizer and analysed with pClamp 11 software (all from Molecular Devices). Recording pipettes were pulled from borosilicate capillary glass (Harvard Apparatus; GC150F-10) with a DMZUniversal-Electrode-Puller (Zeitz) and had resistances between 3 and $4 \mathrm{M} \Omega$

Miniature EPSC (mEPSC) were recorded from primary cultured hippocampal neurons after $48 \mathrm{~h}$ of picrotoxin treatment at DIV $21-$ 23. The extracellular solution was composed of (in mM) $140 \mathrm{NaCl}$, $2.5 \mathrm{KCl}, 10 \mathrm{HEPES}, 2 \mathrm{CaCl}_{2}, 1 \mathrm{MgCl}_{2}, 10$ glucose (adjusted to $\mathrm{pH} 7.3$ with $\mathrm{NaOH}$ ), the intracellular solution of (in mM) $125 \mathrm{~K}$-Gluconate, $20 \mathrm{KCL}, 0.5$ EGTA, 10 HEPES, $4 \mathrm{Mg}$-ATP, $0.3 \mathrm{GTP}$ and $10 \mathrm{Na}_{2}$ Phosphocreatine (adjusted to $\mathrm{pH} 7.3$ with $\mathrm{KOH}$ ). $1 \mu \mathrm{M}$ TTX and $1 \mu \mathrm{M}$ Gabazine were added to the extracellular solution to block action-potential driven glutamate release and GABAergic synaptic transmission, respectively. Cells were held at $-70 \mathrm{mV}$. The sampling frequency was $5 \mathrm{kHz}$ and the filter frequency $2 \mathrm{kHz}$. Series resistance was monitored, and recordings were discarded if the series resistance changed significantly $(\geq 10 \%)$ or exceeded $20 \mathrm{M} \Omega$.

\section{Single molecule fluorescence in situ hybridization}

Dissociated hippocampal neurons were fixed at 20DIV using $4 \%$ paraformaldehyde/4\% sucrose/PBS for $30 \mathrm{~min}$ at room temperature. FISH was performed using the QuantiGene (QG) ViewRNA kit (Affymetrix) according to the manufacturer's protocol using probes for Add2, Dnajc6 and Sort1. The protease treatment step was omitted in to maintain dendritic integrity. After completion of the FISH protocol, cells were processed for immunostaining, using an anti-MAP2 antibody $(1: 1,000$, Sigma Cat. Nr.: M9942, RRID: AB_477256) in GDB buffer $(0.02 \%$ gelatin- $0.5 \%$ Triton X-100PBS). Images were acquired on a Leica SP5 laser-scanning confocal microscope. For z-stack images of whole cells, 12 consecutive optical sections were taken at a $0.4 \mu \mathrm{m}$ interval with a resolution of $1,024 \times 1,024$ pixels using a $63 \times$ objective and a digital zoom factor of 2.5. For images of cell bodies, 15 consecutive optical sections were taken at a $0.4 \mu \mathrm{m}$ interval with a resolution of $512 \times 512$ pixels using a $63 \times$ objective and a digital zoom factor of 5 . For all pictures, the pinhole was set to $1 \mathrm{AU}$. Laser settings were kept constant between experimental conditions. Maximum intensity projections of the z-stacks were used for signal quantification, which was conducted in a blinded manner. The density of RNA particles was analysed by CellProfiler (Carpenter et al, 2006), using the MAP2 immunostaining as mask to define cell bodies and dendrites.

\section{Puromycin-proximity ligation assay}

Detection of translation foci was performed using an antibody against puromycin after metabolic labelling of ribosomes by puromycin and an antibody against the nascent peptide of interest as previously described (tom Dieck et al, 2015). The Duolink in situ orange PLA mouse/rabbit kit (Sigma) allows the spatial coincidence detection $(<40 \mathrm{~nm})$ of these two antibodies via a rolling circle amplification step using fluorescently labelled oligonucleotides. For better background estimation, conditions where cells were left untreated of puromycin but processed with all respective antibodies, were included. In all conditions, cells were incubated with antibodies against puromycin (mouse anti-puromycin [3RH11], 1:2,500, Kerafast, Cat. Nr.: EQ0001, RRID: AB_2620162), the peptide of interest (either rabbit anti-Camk2a, 1:500, Thermo Fisher Scientific, Cat. Nr.: PA5-84083, RRID: AB_2791235, 1:500 or rabbit anti-Synapsin1, 1:500, Sigma, Cat. Nr. AB1543, RRID: AB_2200400) and the cellmarker MAP2 (chicken-anti-MAP2, 1:1,000, Thermo Fisher Scientific, Cat. Nr.: PA1-16751, RRID: AB_2138189).

In brief, primary hippocampal neurons were cultured on coverslips as described earlier and treated with $100 \mathrm{nM}$ PTX for $48 \mathrm{~h}$. Before fixation, cells were incubated with puromycin $(3 \mu \mathrm{M}$ final concentration, Invivogen) for $5 \mathrm{~min}$ or left untreated for control conditions, briefly rinsed twice with PBS and fixed in $4 \%$ sucrose-PFA for $10 \mathrm{~min}$ at room temperature. Cells were then rinsed twice in PBS and incubated in permeabilization buffer $(0.05 \%$ Triton X, $10 \%$ normal goat serum in PBS) for $5 \mathrm{~min}$ at room temperature. After permeabilization, we proceeded with the Duolink Proximity Assay (Sigma) according to the manufacturer's protocol with 
slight modifications. The coverslips were placed on parafilm facing up in the wet-chamber of the RNAscope HybEZ ${ }^{\text {TM }}$ II oven (ACD), where all temperature-sensitive steps were carried out. Cells were first treated with the supplied blocking buffer for $1 \mathrm{~h}$ at $37^{\circ}$ and then immediately incubated with the primary antibody solution (mouse anti-puromycin, rabbit anti-peptide of interest and chicken antiMAP2 in supplied antibody diluent) for $1 \mathrm{~h}$ at room temperature. All subsequent incubation steps were followed by three washing steps of 5 min each in washing buffer A (supplied by the Duolink kit). After washing, cells were incubated in secondary antibody solutions (anti-rabbit Plus-probe, 1:5, anti-mouse Minus-probe, 1:5 and anti-chicken Alexa Plus 647-secondary antibody, 1:1,000, Thermo Fisher Scientific, Cat. Nr: A32933, RRID: AB_2762845) for $1 \mathrm{~h}$ at $37^{\circ} \mathrm{C}$ followed by a ligation step $\left(30 \mathrm{~min}\right.$ at $37^{\circ} \mathrm{C}$ ) and amplification step (100 min at $37^{\circ} \mathrm{C}$ ) according to manufacturer's protocol. The final washing steps consisted of three times 10 min with the supplied washing buffer $\mathrm{B}$, followed by a brief nuclear counterstaining with Hoechst $(1: 10,000)$ in PBS and mounting on a glass slide using Aqua-Poly-Mount (Polysciences, Cat. Nr: 18606-20). Imaging was performed within the next week, and the slides stored at $4^{\circ} \mathrm{C}$ upon further use. Images were acquired in a AiryScan Zeiss LSM 800 in Fast Airyscan optimal high-resolution acquisition mode $(1,920 \times 1,920$ pixels, $Z$-Stacked with an interval of $0.18 \mu \mathrm{m}$ across the whole cells) using a $63 \times 1.4 \mathrm{NA}$ oil objective (Plan-Apochromat $63 \times / 1.4$ Oil DIC M27). After inspection of proper Duolink signal and avoiding the edge of the coverslips, 10 images per condition and replicate were acquired using the MAP2-channel and morphological assessment of the neurons as the only criteria for the selection of cells. Images were post-processed using the Airyscan-Processing function on the Zeiss Zen Blue acquisition programme. A custom Python-script written in the context of the ImageJ-framework (Schindelin et al, 2012) was used for image analysis and is freely available via the ImageJ-update site (for more information https:// github.com/dcolam/Cluster-Analysis-Plugin). In brief, to count the Duolink signal within the somatic compartment, a selection mask was created using the MAP2-channel and the nuclei were detected within to get rid of astrocytic nuclei. The neuronal nuclei were expanded by $3 \mu \mathrm{m}$ and used as a somatic mask to count the Duolink spots within. To create the selection mask for the process compartment, the somatic mask was inverted and used to detect the MAP2channel within the inverted somatic mask. The resulting selection was used as a mask for the process compartment, where the Duolink signal was counted. The count of Duolink signal was normalized by the area of the corresponding selection. The Duolinkdensity was then averaged across biological replicate and condition and $\log _{10}$-transformed.

\section{RNA extraction}

RNA was extracted from primary hippocampal neuronal cultures using either peqGOLD Isolation Systems TriFast $^{\mathrm{TM}}$ (Peqlab) to prepare sequencing library or mirVana ${ }^{\mathrm{TM}}$ total RNA Isolation Kit for quantitative real-time PCR following the manufacturer's protocol. To remove potential DNA contamination, RNA samples were treated with TURBO $^{\mathrm{TM}}$ DNase (Ambion) and RNA was re-extracted as described before. Samples were stored at $-80^{\circ} \mathrm{C}$ until further use. Prior to RNA extraction, compartmentalized cell culture system was pre-processed as described (Zheng et al, 2001).

\section{Quantitative real-time PCR}

RNA was reverse-transcribed with iScript ${ }^{\mathrm{TM}}$ reverse transcription supermix (Bio-Rad) using random hexamers (total RNA) or oligo dT20primers (poly-RNA) according to manufacturer's instructions. Quantitative real-time PCR was performed with the Step One Plus Real-Time PCR System (Applied Biosystems), using iTaq SYBR Green Supermix with ROX (Bio-Rad) for detection of mRNA.

\begin{tabular}{|c|c|}
\hline Primers: & \\
\hline \multirow[t]{2}{*}{ Sort1: } & FW (ATCCACGTGTCAACACACCA) \\
\hline & Rev (CATCCATCAACACCATCTCA) \\
\hline \multirow[t]{2}{*}{ Add2: } & FW (ACAAGGATGGAGGATAGTTCCCA) \\
\hline & Rev (GCCTGTCCAGTCTCTCTCCT) \\
\hline \multirow[t]{2}{*}{ Srcin1: } & FW (AGCGAGATCCGTTTATCGAC) \\
\hline & Rev (GGTTGCTCCTCTCTCATCCT) \\
\hline \multirow[t]{2}{*}{ Shank2: } & FW (AATCGTCGCTATCCCCGGAA) \\
\hline & Rev (GTATCAGCTTTTCCCCCTCG) \\
\hline \multirow[t]{2}{*}{ Dnajc6: } & FW (CGACCGTGGAAAAGGATCTA) \\
\hline & Rev (AGGCCCCTTTTTGTCTTTGT) \\
\hline \multirow[t]{2}{*}{ Plk2: } & FW (AGGATAGCACCATCGGAAGTGT) \\
\hline & ReU (ACTCAAACGACGTCCTCCFCAACTC) \\
\hline \multirow[t]{2}{*}{ Gapdh: } & FW (GCCTTCTCTTGTCACAAAGTCGA) \\
\hline & Reu (CCGTCGGTAGAGTCATACTCGAA) \\
\hline
\end{tabular}

\section{Ribo(-) RNA-sequencing}

Library preparation and sequencing were performed by the Max Planck-Genome-Centre Cologne, Germany (http://mpgc.mpipz. mpg.de/home/) for compartmentalized RNA samples. For the first step, rRNA depletion (Ribo-Zero rRNA removal Kit [Illumina]) has been performed with $1 \mu \mathrm{g}$ total RNA per sample $(n=2$ samples per condition), followed by library preparation with NEBNext Ultra ${ }^{\mathrm{TM}}$ Directional RNA Library Prep Kit for Illumina (New England Biolabs). Sequencing was performed as $100 \mathrm{bp}$ single read sequencing on HiSeq2500 ${ }^{\mathrm{TM}}$ (Illumina).

\section{Protein extraction}

Primary hippocampal compartmentalized cultures were prepared as described earlier and treated at DIV19 with either $100 \mu \mathrm{M}$ PTX or vehicle for $48 \mathrm{~h}$. Inserts were then rinsed twice with ice-cold phosphate buffered saline (PBS, Gibco ${ }^{\mathrm{TM}}$ ) and lysed using a cell scraper in home-made RIPA-buffer $(150 \mathrm{mM} \mathrm{NaCl}, 1 \%$ Triton X100; $0.5 \%$ Sodium Deoxycholate; $1 \mathrm{mM}$ EDTA; $1 \mathrm{mM}$ EGTA; $0.05 \%$ SDS; $50 \mathrm{mM}$ Tris $\mathrm{pH} 8$ ) containing a protease inhibitor cocktail (1:1,000; Roche). Cell lysates were then homogenized by pipetting and centrifuged for $30 \mathrm{~min}$ at $13,000 \mathrm{~g}\left(4^{\circ} \mathrm{C}\right)$. The supernatant was snap-frozen in liquid nitrogen and stored at $-80^{\circ} \mathrm{C}$ until further use. Pierce ${ }^{\mathrm{TM}}$ BCA Protein Assay Kit (Thermo Scientific $^{\mathrm{TM}}$ ) was used to quantify protein concentrations following the manufacturer's protocol. See Appendix for the method description of label-free proteomics. 


\section{Bioinformatic analysis}

\section{RNA-Sequencing analysis}

Adapter sequences were trimmed from the reads using Trimmomatic 0.38 with ILLUMINACLIP 1:30:10, LEADING:5, TRAILING:5, SLIDINGWINDOW:5:15 AVGQUAL:20 and MINLEN:30. The reads were then mapped to the rat genome Rnor 6.0 using Subread subjunc 1.33.16 and the Ensembl 96 transcript annotation, and quantified with featureCounts (v.1.6) (Liao et al, 2014). Counts were then aggregated to gene symbols and only genes with more than 20 reads in at least two samples for further processing.

Counts were then aggregated to gene symbols and only genes with more than 20 reads in at least two samples were considered for further processing.

Differential expression analysis was performed with edgeR (v. 3.28) (Robinson et al, 2010) using TMM normalization. Specifically, linear models were fitted using glmFit through all four conditions $(\sim$ compartment * treatment $)$, and differentially expressed genes were identified by testing for the treatment effect in each compartment using separate contrasts.

For the enrichment analyses, a compartment-specific background of expressed genes was determined by taking only genes with more than 20 reads in at least two conditions within the respective compartment into account.

\section{Proteomics data analysis}

A comprehensive library of detected proteins was generated by combining a search against a $R$. Norvegicus reference proteome with exclusively reviewed entries (Swiss-Prot, UP000002494, 8,104 proteins) with a search against a reference proteome containing also predicted entries (TrEMBL, UniProt UP000002494, 21,838 proteins) if proteins were undetectable in more than two samples. We detected 4,047 proteins with no missing values across all conditions out of the total 4,250 proteins. Values were first normalized by variance stabilizing transformation using the vsn package (version 3.54.0), and missing values were imputed using the MinProb function ( $q$-value $<0.01$ ) of the DEP package (version 1.8.0).

The package limma (version 3.42.2) was used to perform differential expression analysis using a linear multiplicative model fitted through all four conditions ( $\sim$ compartment $*$ treatment), and differential expression was computed using empirical Bayes statistics to contrast treatment and compartment effects.

\section{GO enrichment analysis}

Gene Ontology enrichment analysis was performed using the TopGo algorithm (v.2.40.0) (Alexa et al, 2006), essentially as described previously in (Lackinger et al, 2019).

For the compartment analysis (Fig 1D, Appendix Fig S1), significantly enriched genes with a $\operatorname{logFC}$ of greater than \pm 1 were tested against the respective specific background. Subsequently, the Top 6/10 GO-Terms in each ontology were plotted (with those filtered out that have more than 1,000 annotated genes in $\mathrm{CC}$ or more than 300 annotated genes in BP).

PTX GO-terms were obtained by either testing exclusive gene sets (as characterized in Fig 2C) against the respective specific background (Fig 2D) or by testing all significantly down-/upregulated genes in a specific compartment (Appendix Fig S6). For Fig 2D, the Top 5 obtained GO-Terms of all three ontologies were sorted by
Enrichment and of those, the Top 6 GO-Terms plotted. GO-Terms with more than 1,000 annotated genes in an ontology were filtered out.

GO-Terms enriched in the proteomics dataset were identified by aggregating multiple detected protein isoforms and subsequently performing enrichment analysis of significantly changing proteins (criteria proteomics data analysis) with TopGo against the background of detected proteins. For plotting, GO-terms with more than 500 annotated genes (CC) were filtered out.

\section{miRNA-binding site enrichment analyses}

Predicted conserved MBS of rat conserved miRNA families (Targetscan 7.2) (Agarwal et al, 2015), were counted in exclusive gene sets as described in Fig $2 \mathrm{C}$ and tested for an enrichment against the specific background using a hypergeometric test on the number of binding sites (soon to be made available on Bioconductor).

We considered only those miRNAs, which are considerably expressed in hippocampal neurons at this developmental stage (expressed higher than the median of all detected miRNAs in Rajman et al, 2017).

\section{3'UTR length analysis}

In order to get an estimate of the average $3^{\prime}$ UTR lengths of genes present in somata and processes, genomic coordinates of the longest transcript associated with each detected gene were downloaded in $\mathrm{R}$ (AnnotationHub, Ensemble v.100, Rattus Norvegicus). As long $3^{\prime}$ UTR isoforms are specifically prevalent in the brain (Miura et al, 2013), we chose to focus on these in our analysis.

Only $3^{\prime}$ UTRs with a length of more than 20 nucleotides were considered for the comparisons.

\section{RNA-binding-protein motif analysis}

To identify enriched RBP motifs within $3^{\prime}$ UTRs in differentially expressed genes, we extracted the matching FASTA sequences according to the criteria specified in the $3^{\prime}$ UTR length analysis section. Sequences corresponding to differentially up-/downregulated genes in each compartment upon PTX treatment were submitted to the AME-tool of the meme-suite (McLeay \& Bailey, 2010) in combination with the CISBP database of known RBP motifs (Ray et al, 2013).

In AME, standard parameters were used with the Average Odds Score as scoring method and Fisher's exact test for the enrichment estimation. RBP-names in the plots were obtained by comparing the AME output motif_ID to the CISBP database motif_ID annotations.

\section{Additional bioinformatical analyses}

See Appendix for the method description of comparison to external dataset, Exon-Intron split analysis (EISA) and analysis of half-lives.

\section{Graphical representations}

Plots were generated using Affinity Designer, Cytoscape, BioRender.com \& R (ggplot2 [v.3.3.1], ggpubr [v.0.4.0], ggsci [v.2.9] and ggrepel [v.0.8.1]).

\section{Statistics}

The number of independent experiments is indicated in the plots, if not explicitly specified differently in the methods section. Boxplots represent data as follows: central line: median; box: $25^{\text {th }}$ to $75^{\text {th }}$ percentile; whiskers: at most 1.5 times the interquartile range (IQR: 
distance between the $25^{\text {th }}$ to $75^{\text {th }}$ percentile); point outside: outliers outside the whiskers. Normality was tested using the Shapiro-Wilk test considering a $P$-value under 0.05 non-normally distributed. Normally distributed data were tested using one- or two sample Student's $t$-test (always two-sided) or ANOVA and otherwise for non-normal data the non-parametric counterpart tests MannWhitney $U$-test or Kruskal-Wallis test. Differences in cumulative distributions were assessed by two-sample Kolmogorov-Smirnow test. Bivariate correlation analysis between two variables was evaluated using the non-parametric Spearman rank correlation or the parametric Pearson's correlation using the function ggscatter (from ggpubr).

Additional information about statistical tests not described in the figure legends are provided in the Appendix.

\section{Data accessibility}

RNA-seq data have been deposited to GEO (GSE155540):

https://www.ncbi.nlm.nih.gov/geo/query/acc.cgi?acc = GSE155540

The mass spectrometry proteomics data have been deposited to the ProteomeXchange Consortium via the PRIDE partner repository (PXD020745):

http://www.ebi.ac.uk/pride/archive/projects/PXD020745

The following figures have associated raw data: Figs 1, 2 and 4, Appendix Fig S1-S7. Selected data are available at https://dcolame thins.shinyapps.io/Compartment_PTX_App/.

Expanded View for this article is available online.

\section{Acknowledgements}

We greatly acknowledge the excellent technical assistance provided by Eva Becker and Tatjana Wüst. This work is funded by a grant from the DFC to CD and CS (SCHR 1136/4-2). The laboratory of JB is funded by ETH Project Grant ETH-20 19-1, SNSF Grant 310030_172889/1 and the Botnar Foundation.

\section{Author contributions}

DC performed proteomics, QPCR, Puromycin-PLA-experiments, RNA-seq analysis and generated the figures. MR performed RNA-seq. MS performed motif enrichment analysis. SB and MR performed and analysed the SmFISH data. LVZ and JB supervised the proteomics experiments. JW performed electrophysiology experiments. CD helped with RNA-seq data analysis. P-LG supervised RNAseq and motif enrichment analysis. CS coordinated the project and wrote the manuscript.

\section{Conflict of interest}

The authors declare that they have no conflict of interest.

\section{References}

Agarwal V, Bell GW, Nam JW, Bartel DP (2015) Predicting effective microRNA target sites in mammalian mRNAs. Elife 4: e05005

Alexa A, Rahnenfuhrer J, Lengauer T (2006) Improved scoring of functional groups from gene expression data by decorrelating $\mathrm{GO}$ graph structure. Bioinformatics 22: 1600-1607
Amen AM, Ruiz-Garzon CR, Shi J, Subramanian M, Pham DL, Meffert MK (2017) A rapid induction mechanism for Lin28a in trophic responses. Mol Cell 65: $490-503 . e 497$

Aoto J, Nam Cl, Poon MM, Ting P, Chen L (2008) Synaptic signaling by alltrans retinoic acid in homeostatic synaptic plasticity. Neuron 60: 308-320

Bartel DP (2009) MicroRNAs: target recognition and regulatory functions. Cell 136: $215-233$

Bicker S, Khudayberdiev S, Weiss K, Zocher K, Baumeister S, Schratt G (2013) The DEAH-box helicase DHX36 mediates dendritic localization of the neuronal precursor-microRNA-134. Genes Deu 27: 991 -996

Bottini S, Hamouda-Tekaya N, Mategot R, Zaragosi L-E, Audebert S, Pisano S, Grandjean V, Mauduit C, Benahmed M, Barbry P et al (2017) Posttranscriptional gene silencing mediated by microRNAs is controlled by nucleoplasmic Sfpq. Nat Commun 8: 1189

Cajigas IJ, Tushev G, Will T, tom Dieck S, Fuerst N, Schuman E (2012) The local transcriptome in the synaptic neuropil revealed by deep sequencing and high-resolution imaging. Neuron 74: 453-466

Carpenter AE, Jones TR, Lamprecht MR, Clarke C, Kang IH, Friman O, Guertin DA, Chang JH, Lindquist RA, Moffat J et al (2006) Cell profiler: image analysis software for identifying and quantifying cell phenotypes. Genome Biol 7: R100

Chao Sun AN, Tchumatchenko T, Heilemann M, Schuman EM (2020) The Spatial Scale of synaptic protein allocation during homeostatic plasticity. biorxiu https://doi.org/10.1101/2020.04.29.068833 [PREPRINT]

Desai NS, Cudmore RH, Nelson SB, Turrigiano GG (2002) Critical periods for experience-dependent synaptic scaling in visual cortex. Nat Neurosci 5: $783-789$

tom Dieck S, Kochen L, Hanus C, Heumüller M, Bartnik I, Nassim-Assir B, Merk K, Mosler T, Garg S, Bunse S et al (2015) Direct visualization of newly synthesized target proteins in situ. Nat Methods 12: 411-414

Diering GH, Nirujogi RS, Roth RH, Worley PF, Pandey A, Huganir RL (2017) Homerla drives homeostatic scaling-down of excitatory synapses during sleep. Science 355: $511-515$

Dorrbaum AR, Alvarez-Castelao B, Nassim-Assir B, Langer JD, Schuman EM (2020) Proteome dynamics during homeostatic scaling in cultured neurons. Elife 9: e52939

Epple R, Kruger D, Berulava T, Brehm G, Ninov M, Islam R, Koster S, Fischer A (2021) The coding and small non-coding hippocampal synaptic RNAome. Mol Neurobiol 58: 2940-2953

Fiore R, Rajman M, Schwale C, Bicker S, Antoniou A, Bruehl C, Draguhn A, Schratt G (2014) MiR-134-dependent regulation of Pumilio-2 is necessary for homeostatic synaptic depression. Embo J 33: 2231 - 2246

Folci A, Mapelli L, Sassone J, Prestori F, D’Angelo E, Bassani S, Passafaro M (2014) Loss of hnRNP K impairs synaptic plasticity in hippocampal neurons. J Neurosci 34: 9088-9095

Gerber S, Schratt G, Germain P (2021) Streamlining differential exon and $3^{\prime}$ UTR usage with diffUTR. BMC Bioinformatics 22: 189

Glazewski S, Greenhill S, Fox K (2017) Time-course and mechanisms of homeostatic plasticity in layers $2 / 3$ and 5 of the barrel cortex. Philos Trans $R$ Soc Lond B Biol Sci 372: 20160150

Glock C, Biever A, Tushev G, Bartnik I, Nassim-Assir B, tom Dieck S, Schuman EM (2020) The mRNA translation landscape in the synaptic neuropil. biorxiu https://doi.org/10.1101/2020.06.09.141960 [PREPRINT]

Goold CP, Nicoll RA (2010) Single-cell optogenetic excitation drives homeostatic synaptic depression. Neuron 68: 512-528

Herzog VA, Reichholf B, Neumann T, Rescheneder P, Bhat P, Burkard TR, Wlotzka W, von Haeseler A, Zuber J, Ameres SL (2017) Thiol-linked alkylation of RNA to assess expression dynamics. Nat Methods 14: 1198-1204 
Horvathova I, Voigt F, Kotrys AV, Zhan Y, Artus-Revel CG, Eglinger J, Stadler MB, Giorgetti L, Chao JA (2017) The Dynamics of mRNA turnover revealed by single-molecule imaging in single cells. Mol Cell 68: $615-625 . e 619$

Ibata K, Sun Q, Turrigiano GC (2008) Rapid synaptic scaling induced by changes in postsynaptic firing. Neuron 57: 819-826

Kanai Y, Dohmae N, Hirokawa N (2004) Kinesin transports RNA: isolation and characterization of an RNA-transporting granule. Neuron 43: $513-525$

Kavalali ET, Monteggia LM (2012) Synaptic mechanisms underlying rapid antidepressant action of ketamine. Am J Psychiatry 169: 1150-1156

von Kugelgen N, Chekulaeva M (2020) Conservation of a core neurite transcriptome across neuronal types and species. Wiley Interdiscip Reu RNA 11: e1590

Kuzniewska B, Cysewski D, Wasilewski M, Sakowska P, Milek J, Kulinski TM, Winiarski M, Kozielewicz P, Knapska E, Dadlez M et al (2020) Mitochondrial protein biogenesis in the synapse is supported by local translation. EMBO Rep 21: e48882

Lackinger M, Sungur AO, Daswani R, Soutschek M, Bicker S, Stemmler L, Wüst T, Fiore R, Dieterich C, Schwarting RK et al (2019) A placental mammalspecific microRNA cluster acts as a natural brake for sociability in mice. EMBO Rep 20: e46429

Leal G, Comprido D, de Luca P, Morais E, Rodrigues L, Mele M, Santos AR, Costa RO, Pinto MJ, Patil S et al (2017) The RNA-binding protein hnRNP K mediates the effect of BDNF on dendritic mRNA metabolism and regulates synaptic NMDA receptors in hippocampal neurons. eNeuro 4: ENEURO.0268-17.2017

Liao Y, Smyth GK, Shi W (2014) featureCounts: an efficient general purpose program for assigning sequence reads to genomic features. Bioinformatics 30: $923-930$

Martin KC, Ephrussi A (2009) mRNA localization: gene expression in the spatial dimension. Cell 136: 719-730

McLeay RC, Bailey TL (2010) Motif enrichment analysis: a unified framework and an evaluation on ChIP data. BMC Bioinformatics 11: 165

McNeill EM, Warinner C, Alkins S, Taylor A, Heggeness H, DeLuca TF, Fulga TA, Wall DP, Griffith LC, Van Vactor D (2020) The conserved microRNA miR-34 regulates synaptogenesis via coordination of distinct mechanisms in presynaptic and postsynaptic cells. Nat Commun 11: 1092

Miura P, Shenker S, Andreu-Agullo C, Westholm JO, Lai EC (2013) Widespread and extensive lengthening of $3^{\prime}$ UTRs in the mammalian brain. Genome Res 23: $812-825$

Nagano K, Bornhauser BC, Warnasuriya G, Entwistle A, Cramer R, Lindholm D, Naaby-Hansen S (2006) PDGF regulates the actin cytoskeleton through hnRNP-K-mediated activation of the ubiquitin E3-ligase MIR. Embo J 25: 1871-1882

Poon MM, Choi SH, Jamieson CA, Geschwind DH, Martin KC (2006) Identification of process-localized mRNAs from cultured rodent hippocampal neurons. J Neurosci 26: 13390-13399

Proepper C, Steinestel K, Schmeisser MJ, Heinrich J, Steinestel J, Bockmann J, Liebau S, Boeckers TM (2011) Heterogeneous nuclear ribonucleoprotein $k$ interacts with Abi-1 at postsynaptic sites and modulates dendritic spine morphology. PLoS One 6: e27045

Rabinowitch I, Segev I (2006) The interplay between homeostatic synaptic plasticity and functional dendritic compartments. J Neurophysiol 96: $276-283$

Rabinowitch I, Segev I (2008) Two opposing plasticity mechanisms pulling a single synapse. Trends Neurosci 31: 377-383
Rajman M, Metge F, Fiore R, Khudayberdiev S, Aksoy-Aksel A, Bicker S, Ruedel Reschke C, Raoof R, Brennan GP, Delanty N et al (2017) A microRNA-129$5 \mathrm{p} / \mathrm{Rbfox}$ crosstalk coordinates homeostatic downscaling of excitatory synapses. EMBO J 36: 1770-1787

Ramocki MB, Zoghbi HY (2008) Failure of neuronal homeostasis results in common neuropsychiatric phenotypes. Nature 455: 912-918

Ray D, Kazan H, Cook KB, Weirauch MT, Najafabadi HS, Li X, Gueroussov S, Albu M, Zheng $\mathrm{H}$, Yang A et al (2013) A compendium of RNA-binding motifs for decoding gene regulation. Nature 499: 172-177

Robinson MD, McCarthy DJ, Smyth CK (2010) edgeR: a Bioconductor package for differential expression analysis of digital gene expression data. Bioinformatics 26: 139-140

Sano T, Reynolds JP, Jimenez-Mateos EM, Matsushima S, Taki W, Henshall DC (2012) MicroRNA-34a upregulation during seizure-induced neuronal death. Cell Death Dis 3: e287

Schanzenbacher CT, Sambandan S, Langer JD, Schuman EM (2016) Nascent proteome remodeling following homeostatic scaling at hippocampal synapses. Neuron 92: 358-371

Schanzenbacher CT, Langer JD, Schuman EM (2018) Time- and polaritydependent proteomic changes associated with homeostatic scaling at central synapses. Elife 7: e33322

Schaukowitch K, Reese AL, Kim SK, Kilaru G, Joo JY, Kavalali ET, Kim TK (2017) An intrinsic transcriptional program underlying synaptic scaling during activity suppression. Cell Rep 18: 1512-1526

Schindelin J, Arganda-Carreras I, Frise E, Kaynig V, Longair M, Pietzsch T, Preibisch S, Rueden C, Saalfeld S, Schmid B et al (2012) Fiji: an opensource platform for biological-image analysis. Nat Methods 9: 676-682

Schratt GM, Tuebing F, Nigh EA, Kane CG, Sabatini ME, Kiebler M, Greenberg ME (2006) A brain-specific microRNA regulates dendritic spine development. Nature 439: 283-289

Seeburg DP, Feliu-Mojer M, Gaiottino J, Pak DT, Sheng M (2008) Critical role of CDK5 and Polo-like kinase 2 in homeostatic synaptic plasticity during elevated activity. Neuron 58: 571-583

Shanmugam N, Reddy MA, Natarajan R (2008) Distinct roles of heterogeneous nuclear ribonuclear protein $\mathrm{K}$ and microRNA-16 in cyclooxygenase-2 RNA stability induced by S100b, a ligand of the receptor for advanced glycation end products. J Biol Chem 283: 36221-36233

Sutton MA, Wall NR, Aakalu GN, Schuman EM (2004) Regulation of dendritic protein synthesis by miniature synaptic events. Science 304: 1979-1983

Sutton MA, Ito HT, Cressy P, Kempf C, Woo JC, Schuman EM (2006) Miniature neurotransmission stabilizes synaptic function via tonic suppression of local dendritic protein synthesis. Cell 125: 785 - 799

Swann JW, Rho JM (2014) How is homeostatic plasticity important in epilepsy? Adv Exp Med Biol 813: 123-131

Tononi G, Cirelli C (2014) Sleep and the price of plasticity: from synaptic and cellular homeostasis to memory consolidation and integration. Neuron 81: $12-34$

Turrigiano GC (2008) The self-tuning neuron: synaptic scaling of excitatory synapses. Cell 135: $422-435$

Turrigiano G (2012) Homeostatic synaptic plasticity: local and global mechanisms for stabilizing neuronal function. Cold Spring Harb Perspect Biol 4: a005736

Wayman GA, Davare M, Ando H, Fortin D, Varlamova O, Cheng H-Y, Marks D, Obrietan K, Soderling TR, Goodman RH et al (2008) An activity-regulated microRNA controls dendritic plasticity by down-regulating p250GAP. Proc Natl Acad Sci USA 105: 9093-9098

Zappulo A, van den Bruck D, Ciolli Mattioli C, Franke V, Imami K, McShane E, Moreno-Estelles M, Calviello L, Filipchyk A, Peguero-Sanchez E et al (2017) 
RNA localization is a key determinant of neurite-enriched proteome. Nat Commun 8: 583

Zheng JQ, Kelly TK, Chang B, Ryazantsev S, Rajasekaran AK, Martin KC, Twiss $J \mathrm{~L}$ (2001) A functional role for intra-axonal protein synthesis during axonal regeneration from adult sensory neurons. J Neurosci 21: $9291-9303$

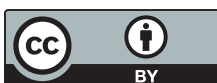

License: This is an open access article under the terms of the Creative Commons Attribution License, which permits use, distribution and reproduction in any medium, provided the original work is properly cited. 